\title{
Fast synchronization of complex dynamical networks with time-varying delay via periodically intermittent control
}

\author{
Yihan Fan ${ }^{\mathrm{a}}$, Hongmei Liu ${ }^{\mathrm{a}}$, Yonggang Zhu ${ }^{\mathrm{a}}$, Jun Mei ${ }^{\mathrm{b}, *}$ \\ ${ }^{a}$ College of Science, China Three Gorges University, Yichang, Hubei 443002, China \\ ${ }^{b}$ Centre of New Energy Systems, Department of Electrical, Electronic and Computer \\ Engineering, University of Pretoria, Pretoria 0002, South Africa
}

\begin{abstract}
The fast synchronization problem for a class of complex dynamical networks with time varying delay by means of periodically intermittent control is studied. Based on the finite-time stability theory and periodically intermittent control technique, some sufficient synchronization criteria are obtained to guarantee the fast synchronization. Furthermore, the essential condition for guaranteeing periodically intermittent control realized in finite time is given in this paper. Finally, two examples are illustrated to verify the proposed theoretical results.
\end{abstract}

Keywords: Complex dynamical networks; fast synchronization; periodically intermittent control; Lyapunov-Krasovskii functional.

\section{Introduction}

Complex networks have recently received great attention in wide fields of science, engineering and society. Networks exist everywhere in nature and our daily life, such as social networks, ecosystems, the internet, World Wide Web, neural networks, and so on. Generally, a complex network can be described by a large set of nodes and edges interconnecting these nodes.

Among various dynamical behaviors, synchronization is a significant and interesting phenomenon. Synchronization plays a very important role in many contexts, such as synchronous communication, signal synchronization (for example, synchronization between video and audio signals), firefly bioluminescence synchronization, geostationary satellites, synchronous motors,

*Corresponding author.

(Hongmei Liu), meij0000@163.com 
database synchronization [1-5]. So far, many effective control approaches have been proposed to achieve synchronization, such as adaptive control [67], feedback control [8-9], pinning control [10-11], impulsive control [12-13] and intermittent control [14-19].

Recently, the discontinuous feedback control approaches, such as impulsive control and intermittent control, have received a lot of focuses since they are practical and easy implementation in engineering such as transportation and communication. Intermittent control has a nonzero control width, while impulsive control is only activating at some isolated instants. On the other hand, as a special form of switching control, intermittent control can be divided into two classes [16]: state-dependent switching rule and time-dependent switching rule. The former implies that the control operation is activated only when the states enter to a certain region which is often pre-given, while the latter rule activates the control only in some finite time intervals, the system evolving freely outside these intervals. Compared with continuous control methods, intermittent control is more efficient because the system output is measured intermittently rather than continuously [17]. In view of these merits, some dynamic systems with or without time delays were discussed by using the intermittent feedback control, and some promising results were achieved [14-21].

Nevertheless, to the best of our knowledge, most researches focus on the asymptotical or exponential synchronization of networks via intermittent control [14-19]. This means that the trajectories of the slave system can reach to the trajectories of the master system over the infinite horizon by using intermittent control method. From a practical point of view, it is a challenging but significant that the synchronization objective is realized in a finite time [22-28]. In order to achieve a convergence time in a given time, finite-time intermittent control methods can work out efficiently. Periodically intermittent feedback control scheme [20] and periodically intermittent adaptive control method [21] are designed to control the complex dynamical networks to achieve finite-time synchronization. This paper gives some new contributions for fast synchronization of systems via intermittent control. Fast synchronization means the convergence time very fast. Fast stability has been firstly reported in Ref. [29]. Different control problems to study fast convergence are given in Refs. [30, 31]. Fast tracking has been applied in Heating, Ventilation and Air Conditioning (HVAC) control systems. To obtain a high thermal comfort, we hope the controller can fast track the setpoint. In order to save energy consumption by the buildings HVAC, the 
intermittent control technique can be adopted, especially the price of electricity is used under time-of-use (TOU) policy. Therefore, fast synchronization of systems via intermittent control is a very interesting topic.

The contribution of this paper is given as follow: firstly, the aim of this paper is to improve the convergence time for caparisoning with the previous work [20-21]; secondly, two types intermittent control methods (from control to non-control and from non-control to control) are designed; what's more, the control width is less than the convergence time, which is to guarantee the finite-time intermittent control, but this important condition has been neglected in [20-21].

The paper is organized as follows. In Section 2, some useful preliminaries, lemmas, assumptions and definition are presented. In Section 3, the fast synchronization criteria for the complex dynamical networks via periodically intermittent control are obtained. Numerical examples are given to demonstrate the effectiveness of the main results in Section 4. Conclusions are finally drawn in Section 5 .

\section{Preliminaries}

Consider a general complex dynamical network consisting of $N$ dynamical nodes with linear couplings, which is described by

$$
\begin{array}{r}
\dot{x}_{i}(t)=f\left(x_{i}(t)\right)+c_{1} \sum_{j=1}^{N} a_{i j} \Gamma_{1} x_{j}(t)+c_{2} \sum_{j=1}^{N} b_{i j} \Gamma_{2} x_{j}(t-\tau(t)), \\
i=1,2, \cdots, N,
\end{array}
$$

where $x_{i}(t)=\left(x_{i 1}(t), x_{i 2}(t), \cdots, x_{i n}(t)\right)^{T} \in R^{n}$ is the state vector of the $i$ th node; $f: R^{n} \rightarrow R^{n}$ is a continuously differential nonlinear vector function; $c_{1}>0$ and $c_{2}>0$ are the non-delay and time-varying delay coupling strengths, respectively. $\Gamma_{1}=\operatorname{diag}\left(\gamma_{1}^{1}, \gamma_{1}^{2}, \ldots, \gamma_{1}^{n}\right)$ and $\Gamma_{2}=\operatorname{diag}\left(\gamma_{2}^{1}, \gamma_{2}^{2}, \ldots, \gamma_{2}^{n}\right)$ are positive definite diagonal matrices, which represent the inner connection matrices between each pair of nodes. $A=\left(a_{i j}\right)_{N \times N}, B=\left(b_{i j}\right)_{N \times N}$ are the non-delay and time-varying delay weight configuration matrices, respectively. The entries $a_{i j}$ is defined as follows: if there is a link from the node $i$ to the node $j(i \neq j)$, then $a_{i j} \neq 0, b_{i j} \neq 0$; otherwise $a_{i j}=0, b_{i j}=0$, and the diagonal elements of matrices $A, B$ are defined as $a_{i i}=-\sum_{j=1, j \neq i}^{N} a_{i j}, b_{i i}=$ $-\sum_{j=1, j \neq i}^{N} b_{i j}$. Since the networks in this paper are direct, $a_{i j} \neq a_{j i}$ and 
$b_{i j} \neq b_{j i}(i, j=1,2, \ldots, N)$. The coupling time-varying delay $\tau(t)$ is a bounded and continuously differentiable function, which means there exist positive constants $\varepsilon$ and $\tau$ satisfying $0 \leq \dot{\tau}(t) \leq \varepsilon<1$ and $0 \leq \tau(t) \leq \tau$.

To realize finite-time synchronization between two coupled complex networks with time-varying delay via periodically intermittent control, we refer to the model (1) as the drive network, and the corresponding response network is given by the following equation:

$$
\begin{array}{r}
\dot{y}_{i}(t)=f\left(y_{i}(t)\right)+c_{1} \sum_{j=1}^{N} a_{i j} \Gamma_{1} y_{j}(t)+c_{2} \sum_{j=1}^{N} b_{i j} \Gamma_{2} y_{j}(t-\tau(t))+u_{i}(t), \\
i=1,2, \cdots, N,
\end{array}
$$

where $y_{i}(t)=\left(y_{i 1}(t), y_{i 2}(t), \ldots, y_{i n}(t)\right)^{T} \in R^{n}$ is the response state vector of the node $i$ of system $(2)$, and $u_{i}(t)(i=1,2, \cdots, N)$ are the intermittent controllers, which are described as follows:

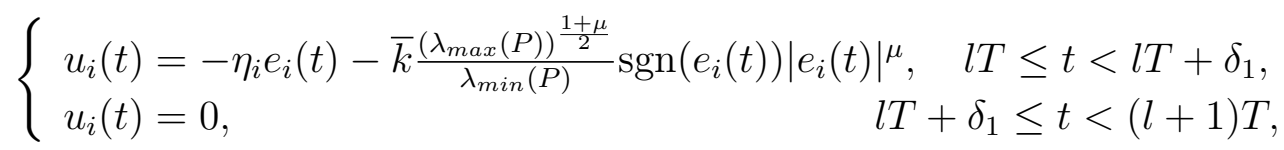

where $\eta_{i}>0$ is a positive constant called control gain; denotes $\lambda_{\max }(P)$ $\left(\lambda_{\min }(P)\right)$ as the maximum (minimum) eigenvalue of the positive definite diagonal matrix $P$, respectively; $\bar{k}>0$ is a tunable real constant; the real number $\mu$ satisfies $0<\mu<1$. $T>0$ is the control period, and $\delta_{1}>0$ is called the control width (control duration). $\theta^{\sim}=\delta_{1} / T$ is the ratio of the control width $\delta_{1}$ to the control period $T$ called control rate, and satisfies $0<\widetilde{\theta}<1$. $\iota=$ $\{1,2, \ldots, \varsigma\}$ is a finite natural number set and $l \in \iota . e_{i}(t)=y_{i}(t)-x_{i}(t), i=$ $1,2, \ldots, N$ are the synchronization errors between the drive network (1) and the response network $(2) ;\left|e_{i}(t)\right|^{\mu}=\left(\left|e_{i 1}(t)\right|^{\mu},\left|e_{i 2}(t)\right|^{\mu}, \ldots,\left|e_{i n}(t)\right|^{\mu}\right)^{T}$; $\operatorname{sgn}\left(e_{i}(t)\right)=\left(\operatorname{sgn}\left(e_{i 1}(t)\right), \operatorname{sgn}\left(e_{i 2}(t)\right), \ldots, \operatorname{sgn}\left(e_{i n}(t)\right)\right)^{T}$, then we have the following error systems

$$
\left\{\begin{aligned}
\dot{e}_{i}(t)= & f\left(e_{i}(t)\right)+c_{1} \sum_{j=1}^{N} a_{i j} \Gamma_{1} e_{j}(t)+c_{2} \sum_{j=1}^{N} b_{i j} \Gamma_{2} e_{j}(t-\tau(t)), \\
& +u_{i}(t), \quad l T \leq t<l T+\widetilde{\theta} T, \quad i=1,2, \cdots, N \\
\dot{e}_{i}(t)= & f\left(e_{i}(t)\right)+c_{1} \sum_{j=1}^{N} a_{i j} \Gamma_{1} e_{j}(t)+c_{2} \sum_{i=1}^{N} b_{i j} \Gamma_{2} e_{j}(t-\tau(t)), \\
& l T+\widetilde{\theta} T \leq t<(l+1) T, \quad i=1,2, \cdots, N
\end{aligned}\right.
$$

Remark 1. There are many control schemes to realize complex networks to get synchronization, such as feedback control, pinning control, adaptive 
control and so on. All the control schedules are based on the continuous closed-loop controllers. Most of practical situations could use the continuous controllers. However, some fields are no need to use the continuous control method, such as microgrid control [32], wind energy conversion control [33], because of controls continuously work are abound to add costs. These controls are intermittent. Hence, it is worth to investigate the intermittent control problem.

In this paper, we give the following definition of finite-time synchronization for the networks (1) and (2).

Definition 1. The systems (1) and (2) are said to achieve local synchronization in finite-time $t^{*}$ if there exists a constant $l$ such that for any solutions of systems (1) and (2) with different initial values $\phi, \varphi \in \Omega=\{\psi \in$ $\left.\mathcal{C}\left(\left[t_{0}-\tau, t_{0}\right], R^{n}\right),\|\psi\|<l\right\}$, and $t^{*}$ depends on the initial state vector values $\phi$ and $\varphi$, for any $t \geq t^{*}$, such that

$$
\left\|y_{i}(t)-x_{i}(t)\right\|=0, \text { as } t \rightarrow t^{*}
$$

holds for any $i=1,2, \ldots, n$, where $x(t)=\left(x_{1}(t), \ldots, x_{n}(t)\right)^{T} \in R^{n}$ and $y(t)=\left(y_{1}(t), \ldots, y_{n}(t)\right)^{T} \in R^{n}$. Furthermore, if $\Omega=\mathcal{C}\left([-\tau, 0], R^{n}\right)$, systems (1) and (2) are said to achieve global synchronization in finite-time $t^{*}$.

In order to obtain the main results, we give the following assumptions and lemmas.

Assumption 1. Assume that there exist a positive definite diagonal matrix $P=\operatorname{diag}\left(p_{1}, \ldots, p_{n}\right)$ and a diagonal matrix $\Theta=\operatorname{diag}\left(\theta_{1}, \ldots, \theta_{n}\right)$, such that $f(\cdot)$ satisfies the following inequality:

$$
(y-x)^{T} P(f(y)-f(x)-\Theta(y-x)) \leq-\xi(y-x)^{T}(y-x),
$$

for some $\xi>0$, all $x, y \in R^{n}$.

The function $f(\cdot) \in$ QUAD $(P, \Theta)$. it can be shown that QUAD assumption holds for several well-known chaotic oscillators, such as the Lorenz's systems, Chua's systems, Rössler's systems, and so on. This assumption has been widely given in the previous work ([10-11], [20-21]).

Assumption 2. There exists a continuous function $g:[0, \infty) \rightarrow[0, \infty)$ with $g(0) \geq 0$, for any $0 \leq u \leq t$, such that

$$
g(t)-g(u) \leq-\lambda \int_{u}^{t}(g(s))^{\rho} \mathrm{d} s,
$$

for any $0<\rho<1$ and $\lambda>0$. 
The following lemmas will play an important role in later analysis, which guarantee the complex dynamical networks achieving fast synchronization by using two types intermittent controller techniques, its initial idea comes from $[20,21]$.

Lemma 1 . Assume that a continuous, positive-definite function $V(t)$ defined on a neighborhood $\widetilde{U} \in R^{n}$ of the origin, and satisfy the following differential inequality:

$$
\dot{V}(x(t)) \leq-\alpha V^{\eta}(x(t))-p V(x(t)), \quad \forall x(t) \in \widetilde{U} \backslash\{0\},
$$

where $\alpha>0,0<\eta<1, p>0$ are constants. Then, for any given $x\left(t_{0}\right), V(t)$ satisfies the following inequality:

$$
\begin{gathered}
V^{1-\eta}(x(t)) \exp \{(1-\eta) p x(t)\} \leq V^{1-\eta}\left(x\left(t_{0}\right)\right) \exp \left\{(1-\eta) p x\left(t_{0}\right)\right\}+\frac{\alpha}{p}[\exp \{ \\
\left.\left.(1-\eta) p x\left(t_{0}\right)\right\}-\exp \{(1-\eta) p x(t)\}\right], \\
t_{0} \leq t \leq t_{s},
\end{gathered}
$$

and

$$
V(x(t)) \equiv 0, \quad \forall t \geq t_{s}
$$

with $t_{s}$ given by

$$
t_{s}=\frac{\ln \left(1+\frac{p}{\alpha} V^{1-\eta}(0)\right)}{p(1-\eta)}
$$

for $t_{0}=0$.

Proof. Consider the following differential equation:

$$
\dot{X}(t)=-\alpha X^{\eta}(t)-p X(t), \quad X\left(t_{0}\right)=V\left(t_{0}\right) .
$$

By multiplying $\exp \{p t\}$, we have

$$
\frac{d(\exp \{p t\} X(t))}{d t}=-\alpha(\exp \{p t\} X(t))^{\eta} \exp \{(1-\eta) p t\} .
$$

Although this differential equation does not satisfy the global Lipschitz condition, the unique solution to this equation can be found as

$$
\begin{aligned}
X^{1-\eta}(t) \exp \{(1-\eta) p t\}= & X^{1-\eta}\left(t_{0}\right) \exp \left\{(1-\eta) p t_{0}\right\}+ \\
& \frac{\alpha}{p}\left[\exp \left\{(1-\eta) p t_{0}\right\}-\exp \{(1-\eta) p t\},\right. \\
& t_{1} \geq t \geq t_{0},
\end{aligned}
$$


and $X(t) \equiv 0, \quad \forall t \geq t_{1}$.

It is direct to prove that $x(t)$ is differential for $t>t_{0}$. From the comparison lemma, one obtains

$$
\begin{array}{r}
V^{1-\eta}(x(t)) \exp \{(1-\eta) p x(t)\} \leq V^{1-\eta}\left(x\left(t_{0}\right)\right) \exp \left\{(1-\eta) p x\left(t_{0}\right)\right\}+\frac{\alpha}{p}[\exp \\
\left.\left\{(1-\eta) p x\left(t_{0}\right)\right\}-\exp \{(1-\eta) p x(t)\}\right], \\
t_{0} \leq t \leq t_{s},
\end{array}
$$

and $V(x(t)) \equiv 0, \quad \forall t \geq t_{s}$, with $t_{s}$ given in (9).

Lemma 2. Suppose that function $V(t)$ is continuous and non-negative when $t \in[0, \infty)$ and satisfy the following conditions:

$$
\begin{cases}\dot{V}(t) \leq-\alpha V^{\eta}(t)-p_{1} V(t), & l T \leq t<l T+\theta T \\ \dot{V}(t) \leq p_{2} V(t), & l T+\theta T \leq t<(l+1) T,\end{cases}
$$

where $\alpha, T, p_{1}, p_{2}>0,0<\eta, \theta<1, l \in \iota$, if

$$
\widetilde{\theta} p_{1}-(1-\theta) p_{2}>0,
$$

then the following inequality holds:

$$
\begin{aligned}
& V^{1-\eta}(t) \exp \left\{(1-\eta) p_{1} t\right\} \\
\leq & \exp \left\{(1-\eta)\left(p_{1}+p_{2}\right)(1-\theta) t\right\}\left[V^{1-\eta}(0)-\right. \\
& \left.\frac{\alpha}{p_{1}}\left(\exp \left\{(1-\eta) p_{1} \theta t\right\} \exp \left\{-(1-\eta) p_{2}(1-\theta) t\right\}-1\right)\right], \quad t \geq 0 .
\end{aligned}
$$

Remark 2. Lemma 2 is given to investigate fast synchronization of complex dynamical networks via the intermittent controllers (3).

Lemma 3 ( Jesen inequality [34]). If $a_{1}, a_{2}, \cdots, a_{n}$ are any positive numbers and $0<r<p$, then

$$
\left(\sum_{i=1}^{n} a_{i}^{p}\right)^{1 / p} \leq\left(\sum_{i=1}^{n} a_{i}^{r}\right)^{1 / r} .
$$

Proposition 1: Suppose that there exists a continuous differential function $H\left(\beta_{1}, \beta_{2}\right)=\frac{\ln \left(1+\frac{\beta_{1} \frac{1-\mu}{2}}{2 \bar{k}}(0)\right.}{\frac{1-\mu}{2}\left(\theta \beta_{1}-(1-\theta) \beta_{2}\right)}$ with any positive constants $1>\mu>$ 
$0, \bar{k}, 1>\theta>0, V(0)$ and $\beta_{1}, \beta_{2} \in(0,+\infty), \theta \beta_{1}-(1-\theta) \beta_{2}>0$. Then $\frac{\partial H}{\partial \beta_{1}}<0, \frac{\partial H}{\partial \beta_{2}}>0$.

Proof: Calculating the derivative of $H\left(\beta_{1}, \beta_{2}\right)$ with $\beta_{1}$, we have

$$
\frac{\partial H}{\partial \beta_{1}}=\frac{\frac{V^{\frac{1-\mu}{2}}(0)(1-\mu)\left(\theta \beta_{1}-(1-\theta) \beta_{2}\right)}{4 \bar{k}\left(1+\frac{\beta_{1} V^{\frac{1-\mu}{2}}(0)}{2 \bar{k}}\right)}-\ln \left(1+\frac{\beta_{1} V^{\frac{1-\mu}{2}}(0)}{2 \bar{k}}\right) \frac{(1-\mu) \theta}{2}}{\left(\frac{1-\mu}{2}\right)^{2}\left(\theta \beta_{1}-(1-\theta) \beta_{2}\right)^{2}} .
$$

Denote $F\left(\beta_{1}, \beta_{2}\right)=\frac{V^{\frac{1-\mu}{2}}(0)(1-\mu)\left(\theta \beta_{1}-(1-\theta) \beta_{2}\right)}{4 \bar{k}\left(1+\frac{\beta_{1} V^{\frac{1-\mu}{2}}}{2 \bar{k}}(0)\right.}-\ln \left(1+\frac{\beta_{1} V^{\frac{1-\mu}{2}}(0)}{2 \bar{k}}\right) \frac{(1-\mu) \theta}{2}, F(0)=$ 0 , the derivative of $F\left(\beta_{1}, \beta_{2}\right)$ with respect to $\beta_{1}$ is given as follows:

$$
\frac{\partial F}{\partial \beta_{1}}=-\frac{2 V^{1-\mu}(0)(1-\mu) \theta_{1} \beta_{1}^{2}}{\left(4 \bar{k}+2 \beta_{1} V^{\frac{1-\mu}{2}}(0)\right)^{2}}<0 .
$$

Therefore, $\frac{\partial H}{\partial \beta_{1}}<0$.

By differentiating the $H\left(\beta_{1}, \beta_{2}\right)$ with $\beta_{2}$, we get

$$
\frac{\partial H}{\partial \beta_{2}}=\frac{(1-\theta) \ln \left(1+\frac{\beta_{1} V^{\frac{1-\mu}{2}}(0)}{2 \bar{k}}\right)}{\frac{1-\mu}{2}\left(\theta \beta_{1}-(1-\theta) \beta_{2}\right)^{2}}>0 .
$$

This completes the proposition.

\section{Fast synchronization criteria}

In this section, we address the finite-time synchronization problems by means of finite-time theory and Lyapunov-based method. Two different intermittent control techniques are designed to control networks for achieving fast synchronization.

Type 1. Using intermittent controllers (3).

Theorem 1. Suppose that Assumptions 1 and 2 hold, there exist positive constants $\eta_{1}, \eta_{2}, \ldots, \eta_{N}, \xi, p_{1}, p_{2}$ and a positive definite diagonal matrix $P>0$ such that the following conditions hold:

$$
\left[\begin{array}{cc}
c_{1}\left(A \otimes P \Gamma_{1}\right)+\left(I_{N} \otimes P \Theta\right)+\left(\frac{c_{2} \exp \{p \tau\}}{2(1-\varepsilon)}-\xi\right. & \\
\left.-\frac{\bar{k}}{\lambda}\right)\left(I_{N} \otimes I_{n}\right)-c_{1}(\Xi \otimes P)+\frac{p}{2}\left(I_{N} \otimes P\right) & \frac{c_{2}}{2}\left(B \otimes P \Gamma_{2}\right) \\
* & -\left(\frac{c_{2}}{2}-\frac{\bar{k}}{\lambda}\right)\left(I_{N} \otimes I_{n}\right)
\end{array}\right]<0,
$$




$$
\begin{gathered}
{\left[\begin{array}{cr}
\left(-\xi+\frac{c_{2} \exp \{p \tau\}}{2(1-\varepsilon)}\right)\left(I_{N} \otimes I_{n}\right)+\left(I_{N} \otimes P \Theta\right)+ & \\
c_{1}\left(A \otimes P \Gamma_{1}\right)-\frac{q}{2}\left(I_{N} \otimes P\right) & \frac{c_{2}}{2}\left(B \otimes P \Gamma_{2}\right) \\
* & -\frac{c_{2}}{2}\left(I_{N} \otimes I_{n}\right)
\end{array}\right]<0,} \\
\tilde{\theta} p-(1-\tilde{\theta}) q>0, \\
\tilde{\theta}<\min \left\{1, \frac{q}{p+q}+\sqrt{\left.\frac{\ln \left(1+\frac{p V^{\frac{1-\mu}{2}}(0)}{\alpha}\right)}{\frac{1-\mu}{2} T(p+q)}+\left(\frac{q}{2(p+q)}\right)^{2}\right\}}\right.
\end{gathered}
$$

where $\Xi=\operatorname{diag}\left(\eta_{1}, \eta_{2}, \ldots, \eta_{N}\right), \Theta=\operatorname{diag}\left(\theta_{1}, \theta_{2}, \ldots, \theta_{n}\right)$ and $I_{N}$ is the $N \times N$ identity matrix. Then under the periodically intermittent controllers (3), the error systems (4) can converge to zero in a finite time

$$
t \leq \frac{\ln \left(1+\frac{p V^{\frac{1-\mu}{2}}(0)}{\alpha}\right)}{\frac{1-\mu}{2}(\widetilde{\theta} p-(1-\widetilde{\theta}) q)}=T_{1}
$$

where $V(0)=\sum_{i=1}^{N} e_{i}^{T}(0) P e_{i}(0)+\frac{c_{2} \exp \{p \tau\}}{2(1-\varepsilon)} \sum_{i=1}^{N} f_{-(0)}^{\beta} \exp \{p s\} e_{i}^{T}(s) e_{i}(s) \mathrm{d} s$,
$e_{i}(0)$ is the initial condition of $e_{i}(t)$.

Proof. Define the Lyapunov function as

$$
V(t)=V_{1}(t)+V_{2}(t)
$$

where

$$
\begin{gathered}
V_{1}(t)=\frac{1}{2} \sum_{i=1}^{N} e_{i}^{T}(t) P e_{i}(t), \\
V_{2}(t)=\frac{c_{2} \exp \{p \tau\}}{2(1-\varepsilon)} \sum_{i=1}^{N} \int_{t-\tau(t)}^{t} \exp \{p(s-t)\} e_{i}^{T}(s) e_{i}(s) \mathrm{d} s .
\end{gathered}
$$

The derivative of (22) with respect to time along with the solution of (4) is calculated as follows.

Case I: When $l T \leq t<(l+\widetilde{\theta}) T$, for $l \in \iota$,

$$
\dot{V}_{1}(t)=-p V_{1}(t)+\frac{p}{2} \sum_{i=1}^{N} e_{i}^{T}(t) P e_{i}(t)+\sum_{i=1}^{N} e_{i}^{T}(t) P \dot{e}_{i}(t),
$$




$$
\begin{aligned}
\dot{V}_{2}(t)= & -p V_{2}(t)+\frac{c_{2} \exp \{p \tau\}}{2(1-\varepsilon)} \sum_{i=1}^{N}\left[e_{i}^{T}(t) e_{i}(t)-\right. \\
& \left.\exp \{-p \tau(t)\}(1-\dot{\tau}(t)) e_{i}^{T}(t-\tau(t)) e_{i}(t-\tau(t))\right] \\
\leq & -p V_{2}(t)+\frac{c_{2} \exp \{p \tau\}}{2(1-\varepsilon)} \sum_{i=1}^{N}\left[e_{i}^{T}(t) e_{i}(t)-\right. \\
& \left.\exp \{-p \tau\}(1-\varepsilon) e_{i}^{T}(t-\tau(t)) e_{i}(t-\tau(t))\right]
\end{aligned}
$$

Then, it follow from (25), (26), and Assumption 1 that

$$
\begin{aligned}
& \dot{V}(t) \leq-p V(t)+\frac{p}{2} \sum_{i=1}^{N} e_{i}^{T}(t) P e_{i}(t)+\sum_{i=1}^{N} e_{i}^{T}(t) P \dot{e}_{i}(t)+\frac{c_{2} \exp \{p \tau\}}{2(1-\varepsilon)} \sum_{i=1}^{N}[ \\
&\left.e_{i}^{T}(t) e_{i}(t)-\exp \{-p \tau\}(1-\varepsilon) e_{i}^{T}(t-\tau(t)) e_{i}(t-\tau(t))\right] \\
& \leq \sum_{i=1}^{N}\left\{e_{i}^{T}(t) P\left[f\left(e_{i}(t)\right)-\Theta e_{i}(t)\right]+e_{i}^{T}(t) P \Theta e_{i}(t)+\right. \\
& c_{1} \sum_{j=1}^{N} e_{i}^{T}(t) a_{i j} P \Gamma_{1} e_{j}(t)+c_{2} \sum_{j=1}^{N} e_{i}^{T}(t) b_{i j} P \Gamma_{2} e_{j}(t-\tau(t))+ \\
&\left.\frac{p}{2} e_{i}^{T}(t) P e_{i}(t)+e_{i}^{T}(t) P u_{i}(t)\right\}+\frac{c_{2} \exp \{p \tau\}}{2(1-\varepsilon)} \sum_{i=1}^{N} e_{i}^{T}(t) e_{i}(t)- \\
& \frac{c_{2}}{2} \sum_{i=1}^{N} e_{i}^{T}(t-\tau(t)) e_{i}(t-\tau(t))-p V(t) \\
& \leq-p V(t)-\xi \sum_{i=1}^{N} e_{i}^{T}(t) e_{i}(t)+c_{2} \sum_{i=1}^{N} \sum_{j=1}^{N} e_{i}^{T}(t) b_{i j} P \Gamma_{2} e_{j}(t-\tau(t))+ \\
& \frac{c_{1}}{\sum_{i=1}^{N}} \sum_{j=1}^{N} e_{i}^{T}(t) a_{i j} P \Gamma_{1} e_{j}(t)+\frac{c_{2} \exp \{p \tau\}}{2(1-\varepsilon)} \sum_{i=1}^{N} e_{i}^{T}(t) e_{i}(t)- \\
& \quad \frac{c_{2}}{2} \sum_{i=1}^{N} e_{i}^{T}(t-\tau(t)) e_{i}(t-\tau(t))-c_{1} \sum_{i=1}^{N} e_{i}^{T}(t) \eta_{i} P e_{i}(t)+ \\
& \sum_{i=1}^{N} e_{i}^{T}(t) P \Theta e_{i}(t)-\bar{k} \sum_{i=1}^{N} e_{i}^{T}(t) P \operatorname{sgn}\left(e_{i}(t)\right)\left|e_{i}(t)\right|^{\mu}
\end{aligned}
$$


Let $e(t)=\left(e_{1}^{T}(t), e_{2}^{T}(t), \ldots, e_{N}^{T}(t)\right)^{T}$, and we get

$$
\begin{gathered}
c_{1} \sum_{i=1}^{N} \sum_{j=1}^{N} e_{i}^{T}(t) a_{i j} P \Gamma_{1} e_{j}(t)=c_{1} e^{T}(t)\left(A \otimes P \Gamma_{1}\right) e(t), \\
c_{2} \sum_{i=1}^{N} \sum_{j=1}^{N} e_{i}^{T}(t) b_{i j} P \Gamma_{2} e_{j}(t-\tau(t))=c_{2} e^{T}(t)\left(B \otimes P \Gamma_{2}\right) e(t-\tau(t)), \\
c_{1} \sum_{i=1}^{N} e_{i}^{T}(t) \eta_{i} P e_{i}(t)=c_{1} e^{T}(t)(\Xi \otimes P) e(t), \\
\xi \sum_{i=1}^{N} e_{i}^{T}(t) e_{i}(t)=\xi e^{T}(t)\left(I_{N} \otimes I_{n}\right) e(t) .
\end{gathered}
$$

Let $\lambda>0$, from Assumption 2 and Lemma 3, we have

$$
\begin{aligned}
& \frac{\bar{k}}{\lambda}\left[\sum_{i=1}^{N} e_{i}^{T}(t) e_{i}(t)-\sum_{i=1}^{N} e_{i}^{T}(t-\tau(t)) e_{i}(t-\tau(t))\right] \\
& \leq-\bar{k} \sum_{i=1}^{N} \int_{t-\tau(t)}^{t}\left(e_{i}^{T}(s) e_{i}(s)\right)^{\frac{1+\mu}{2}} \mathrm{~d} s \\
& \leq-\bar{k}\left(\sum_{i=1}^{N} \int_{t-\tau(t)}^{t} e_{i}^{T}(s) e_{i}(s) \mathrm{d} s\right)^{\frac{1+\mu}{2}} .
\end{aligned}
$$

Since $\sum_{i=1}^{N}\left|e_{i}(t)\right|^{T}\left|e_{i}(t)\right|^{\mu}=\sum_{i=1}^{N} \sum_{j=1}^{n}\left|e_{i j}(t)\right|^{1+\mu}$ and using Lemma 3, it implied that

$$
\left(\sum_{i=1}^{N} \sum_{j=1}^{n}\left|e_{i j}(t)\right|^{\mu+1}\right)^{\frac{1}{1+\mu}} \geq\left(\sum_{i=1}^{N} \sum_{j=1}^{n}\left|e_{i j}(t)\right|^{2}\right)^{\frac{1}{2}}
$$

Hence,

$$
\sum_{i=1}^{N} \sum_{j=1}^{n}\left|e_{i j}(t)\right|^{1+\mu} \geq\left(\sum_{i=1}^{N} \sum_{j=1}^{n}\left|e_{i j}(t)\right|^{2}\right)^{\frac{1+\mu}{2}}=\left(\sum_{i=1}^{N} e_{i}^{T}(t) e_{i}(t)\right)^{\frac{1+\mu}{2}}
$$


Therefore, substitutings (28)-(34) into (27), we have

$$
\begin{aligned}
\dot{V}(t) \leq & -p V(t)-\bar{k}\left(\sum_{i=1}^{N} e_{i}^{T}(t) P e_{i}(t)\right)^{\frac{1+\mu}{2}}-\bar{k}\left(\sum_{i=1}^{N} \int_{t-\tau(t)}^{t} e_{i}^{T}(s) e_{i}(s) \mathrm{d} s\right)^{\frac{1+\mu}{2}} \\
& +e^{T}(t)\left[c_{1}\left(A \otimes P \Gamma_{1}\right)+\left(\frac{c_{2} \exp \{p \tau\}}{2(1-\varepsilon)}-\frac{\bar{k}}{\lambda}-\xi\right)\left(I_{N} \otimes I_{n}\right)-\right. \\
& \left.c_{1}(\Xi \otimes P)+\left(I_{N} \otimes P \Theta\right)+\frac{p}{2}\left(I_{N} \otimes P\right)\right] e(t)+c_{2} e^{T}(t)\left(B \otimes P \Gamma_{2}\right) \\
& \left.c_{1}(\Xi \otimes P)+\left(I_{N} \otimes P \Theta\right)+\frac{p}{2}\left(I_{N} \otimes P\right)\right] e(t)+c_{2} e^{T}(t)\left(B \otimes P \Gamma_{2}\right) \\
& e(t-\tau(t))-\left(\frac{c_{2}}{2}-\frac{\bar{k}}{\lambda}\right) e^{T}(t-\tau(t))\left(I_{N} \otimes I_{n}\right) e(t-\tau(t)) \\
\leq & -p V(t)-\bar{k}\left(\sum_{i=1}^{N} e_{i}^{T}(t) e_{i}(t)\right)^{\frac{1+\mu}{2}}-\bar{k}\left(\sum_{i=1}^{N} \int_{t-\tau(t)}^{t} e_{i}^{T}(s) e_{i}(s) \mathrm{d} s\right)^{\frac{1+\mu}{2}} \\
= & -p V(t)-2^{\frac{1+\mu}{2}} \bar{k}\left(\frac{1}{2} \sum_{i=1}^{N} e_{i}^{T}(t) e_{i}(t)\right)^{\frac{1+\mu}{2}}-\bar{k}\left(\frac{c_{2} \exp \{p \tau\}}{2(1-\varepsilon)}\right)^{-\frac{1+\mu}{2}} \\
& \left(\frac{c_{2} \exp \{p \tau\}}{2(1-\varepsilon)} \sum_{i=1}^{N} \int_{t-\tau(t)}^{t} e_{i}^{T}(s) e_{i}(s) \mathrm{d} s\right)^{\frac{1+\mu}{2}} \\
\leq & -p V(t)-\alpha V^{\frac{1+\mu}{2}}(t),
\end{aligned}
$$

where $\alpha=\min \left\{2^{\frac{1+\mu}{2}} \bar{k},\left(\frac{c_{2} \exp \{p \tau\}}{2(1-\varepsilon)}\right)^{-\frac{1+\mu}{2}} \bar{k}\right\}$. Then, $\dot{V}(t) \leq-\alpha V^{\frac{1+\mu}{2}}(t)-p V(t)$.

Case 2: When $(l+\widetilde{\theta}) T \leq t<(l+1) T$, for $l \in \iota$, we can do the similar estimation as the case $l T \leq t<(l+\theta) T$. Thus from Assumption 1, we have

$$
\begin{aligned}
\dot{V}(t)= & -p V(t)+(p+q) V_{1}(t)+\sum_{i=1}^{N} e_{i}^{T}(t) P \dot{e}_{i}(t)+\frac{c_{2} \exp \{p \tau\}}{2(1-\varepsilon)} \sum_{i=1}^{N}\left[e_{i}^{T}(t) e_{i}(t)\right. \\
& \left.-\exp \{-p \tau\}(1-\dot{\tau}(t)) e_{i}^{T}(t-\tau(t)) e_{i}(t-\tau(t))\right]-\frac{q}{2} \sum_{i=1}^{N} e_{i}^{T}(t) P e_{i}(t) \\
\leq & \sum_{i=1}^{N}\left\{e_{i}^{T}(t) P\left[f\left(e_{i}(t)\right)-\Theta e_{i}(t)\right]+e_{i}^{T}(t) P \Theta e_{i}(t)-\frac{q}{2} \sum_{i=1}^{N} e_{i}^{T}(t) P e_{i}(t)+\right. \\
& \left.c_{1} \sum_{j=1}^{N} e_{i}^{T}(t) a_{i j} P \Gamma_{1} e_{j}(t)+c_{2} \sum_{j=1}^{N} e_{i}^{T}(t) b_{i j} P \Gamma_{2} e_{j}(t-\tau(t))\right\}+(p+q) V(t)
\end{aligned}
$$




$$
\begin{aligned}
& +\frac{c_{2} \exp \{p \tau\}}{2(1-\varepsilon)} \sum_{i=1}^{N} e_{i}^{T}(t) e_{i}(t)-\frac{c_{2}}{2} \sum_{i=1}^{N} e_{i}^{T}(t-\tau(t)) e_{i}(t-\tau(t))-p V(t) \\
\leq & -\xi e^{T}(t)\left(I_{N} \otimes I_{n}\right) e(t)+e^{T}(t)\left(I_{N} \otimes P \Theta\right) e(t)+c_{1} e^{T}(t)\left(A \otimes P \Gamma_{1}\right) e(t) \\
& +c_{2} e^{T}(t)\left(B \otimes P \Gamma_{2}\right) e(t-\tau(t))+\frac{c_{2} \exp \{p \tau\}}{2(1-\varepsilon)} e^{T}(t)\left(I_{N} \otimes I_{n}\right) e(t)- \\
& \frac{c_{2}}{2} e^{T}(t-\tau(t))\left(I_{N} \otimes I_{n}\right) e(t-\tau(t))-\frac{q}{2} e^{T}(t)\left(I_{N} \otimes P\right) e(t)+q V(t) \\
\leq & q V(t),
\end{aligned}
$$

which represents that $\dot{V}(t) \leq q V(t)$.

Namely, denotes $\frac{1+\mu}{2}=\eta$, we have

$$
\begin{cases}\dot{V}(t) \leq-\alpha V^{\eta}(t)-p V(t), & l T \leq t<l T+\widetilde{\theta} T \\ \dot{V}(t) \leq q V(t), & l T+\widetilde{\theta} T \leq t<(l+1) T\end{cases}
$$

Using Lemma 2, we obtain

$$
\begin{aligned}
& V^{\frac{1-\mu}{2}}(t) \exp \left\{\frac{1-\mu}{2} p t\right\} \leq \exp \left\{\frac{1-\mu}{2}(p+q)(1-\widetilde{\theta}) t\right\}\left[V^{1-\eta}(0)\right. \\
&\left.-\frac{\alpha}{p}\left(\exp \left\{\frac{1-\mu}{2}(p \widetilde{\theta}-q(1-\widetilde{\theta})) t\right\}-1\right)\right], \\
& t \geq 0 .
\end{aligned}
$$

By Lemma 1, we have

$$
t \leq \frac{\ln \left(1+\frac{p V^{\frac{1-\mu}{2}}(0)}{\alpha}\right)}{\frac{1-\mu}{2}(p \widetilde{\theta}-q(1-\widetilde{\theta}))}
$$

The proof of Theorem 1 is completed.

Remark 3. Inq. (20) should be given for achieving finite-time synchronization via periodically intermittent control. If $1>\widetilde{\theta}>\frac{q}{p+q}+$ $\sqrt{\frac{\ln \left(1+\frac{p V^{\frac{1-\mu}{2}}(0)}{\alpha}\right)}{\frac{1-\mu}{2} T(p+q)}+\left(\frac{q}{2(p+q)}\right)^{2}}$, the error systems (4) would be finite-time syn-
chronized via continuous control, while this trivial case have been investigated 
by many previous works $[8,24-27,29]$. Hence, this case is not discussed in this paper.

Remark 4. From (21), we can easily see the role of the control rate $\widetilde{\theta}$, the tunable constant $\bar{k}$, the constant $\mu$ and the parameters $p, q$ on finite-timely synchronizing error systems (4), and the parameters $\widetilde{\theta}, \bar{k}, \mu, p, q$ are the decision variables of the convergence time. The role of the parameters $\mu, \bar{k}$ have been discussed by Ref. [26], and the convergence time determining by the parameter $\tilde{\theta}$ has been studied by Refs. [20-21]. In this paper, we will focus on the decision variables $p, q$ influences the convergence time. Denotes $T(p, q)=\frac{\ln \left(1+\frac{p V^{\frac{1-\mu}{2}}(0)}{\alpha}\right)}{\frac{1-\mu}{2}(p \widetilde{\theta}-q(1-\widetilde{\theta}))}$, from Proposition 1, it yields $\frac{\partial T}{\partial p}<0$, we follow that $T(p)$ is the strictly monotone decreasing function for the variable $p$, and we obtain that the function $T(p, q)$ is the strictly monotone increasing function with the variable $q$. Hence, the larger the parameter $p$ is, the shorter the convergence time is achieved; the larger the parameter $q$ is, the longer for the convergence time will be.

Remark 5. The convergence time of (21) is shorter than the convergence time of Refs. [20-21]. This is the advantage of my proposed control scheme for the fast synchronization.

Obviously, when $\theta=1$, the intermittent control (3) is degenerated to a continuous control. This trivial case is discussed as follows.

Corollary 1. Suppose that Assumptions 1 and 2 hold, there exist positive constants $\eta_{1}, \eta_{2}, \ldots, \eta_{N}, \xi, p$ and a positive definite diagonal matrix $P>0$ such that the following conditions hold:

$$
\left[\begin{array}{cc}
c_{1}\left(A \otimes P \Gamma_{1}\right)+\left(I_{N} \otimes P \Theta\right)+\left(\frac{c_{2} \exp \{p \tau\}}{2(1-\varepsilon)}-\xi\right. & \\
\left.-\frac{\bar{k}}{\lambda}\right)\left(I_{N} \otimes I_{n}\right)-c_{1}(\Xi \otimes P)+\frac{p}{2}\left(I_{N} \otimes P\right) & \frac{c_{2}}{2}\left(B \otimes P \Gamma_{2}\right) \\
* & -\left(\frac{c_{2}}{2}-\frac{\bar{k}}{\lambda}\right)\left(I_{N} \otimes I_{n}\right)
\end{array}\right]<0,
$$

where $\Xi=\operatorname{diag}\left(\eta_{1}, \eta_{2}, \ldots, \eta_{N}\right), \Theta=\operatorname{diag}\left(\theta_{1}, \theta_{2}, \ldots, \theta_{n}\right)$ and $I_{N}$ is the $N \times N$ identity matrix. Then under the periodically intermittent controllers (3), the error systems (4) can achieve synchronization in a finite time

$$
t \leq \frac{\ln \left(1+\frac{p V^{\frac{1-\mu}{2}}(0)}{\alpha}\right)}{\frac{1-\mu}{2} p}=T_{2},
$$

where $V(0)=\sum_{i=1}^{N} e_{i}^{T}(0) P e_{i}(0)+\frac{c_{2} \exp \{p \tau\}}{2(1-\varepsilon)} \sum_{i=1}^{N} \int_{-\tau(0)}^{0} \exp \{p s\} e_{i}^{T}(s) e_{i}(s) \mathrm{d} s$, $e_{i}(0)$ is the initial condition of $e_{i}(t)$. 
Remark 6. If Lyapunov function $\dot{V}(t) \leq-\alpha V(t)(p=0)$ when controllers are added into the network. Then, this case has been discussed by Ref. [21]. If Lyapunov function $\dot{V}(t) \leq-\alpha V(t)(p=0)$ when controllers are added into the network and the control rate $\theta=1$. Many previous results focus on this case (see [8, 24-27]).

Type 2. Using intermittent controllers (39).

In the following, we will design the other intermittent controllers (39) to achieve the fast synchronization, which are described as follows:

$$
\left\{\begin{array}{l}
u_{i}(t)=0, \\
u_{i}(t)=-\eta_{i} e_{i}(t)-\bar{k} \frac{\left(\lambda_{\max }(P)\right)^{\frac{1+\mu}{2}}}{\lambda_{\min }(P)} \operatorname{sgn}\left(e_{i}(t)\right)\left|e_{i}(t)\right|^{\mu}, \\
l T+\delta_{2} \leq t<(l+1) T,
\end{array}\right.
$$

for $i=1,2 \ldots, N, \delta_{2}$ is the non-control width. $\bar{\theta}=\delta_{2} / T$ is the ratio of the non-control width $\delta_{2}$ to the control period $T$ called non-control rate, and satisfies $0<\bar{\theta}<1$. Where all the other parameters are the same as (3). In this case, the error systems can be rewritten as follows:

$$
\left\{\begin{array}{c}
\dot{e}_{i}(t)=f\left(e_{i}(t)\right)+c_{1} \sum_{j=1}^{N} a_{i j} \Gamma_{1} e_{j}(t)+c_{2} \sum_{j=1}^{N} b_{i j} \Gamma_{2} e_{j}(t-\tau(t)), \\
l T \leq t<l T+\bar{\theta} T, \quad i=1,2, \cdots, N, \\
\dot{e}_{i}(t)=f\left(e_{i}(t)\right)+c_{1} \sum_{j=1}^{N} a_{i j} \Gamma_{1} e_{j}(t)+c_{2} \sum_{j=1}^{N} b_{i j} \Gamma_{2} e_{j}(t-\tau(t)), \\
+u_{i}(t), \quad l T+\bar{\theta} T \leq t<(l+1) T, \quad i=1,2, \cdots, N,
\end{array}\right.
$$

where $f(\cdot)$ is the same as (4) and satisfies Assumption 1.

Remark 7. The above intermittent controllers (3) are about the control time in the first part of a control period. The simplified diagram of this control is given in Fig.1 of Ref. [21]. The intermittent controllers (39) is difference from (3), which designed is about the non-control time in the first part of a control period. The control architecture shows in the following Figure 1. On the other hand, the convergence time of the two types are differences. The two types controllers have the same control principle, but the work time are differences. Besides, no advantages for caparisoning with each other. In order to save energy consumptions and costs, many control systems employ the intermittent control techniques. The type 1 intermittent control schedule can be adopted to track indoor air setpoints [35, 36]. However, electricity rate is a time-of-use in one day in U.S., the type 2 intermittent control schedule is proposed to regulate indoor temperature setpoint at different time zones to reduce costs [37]. 


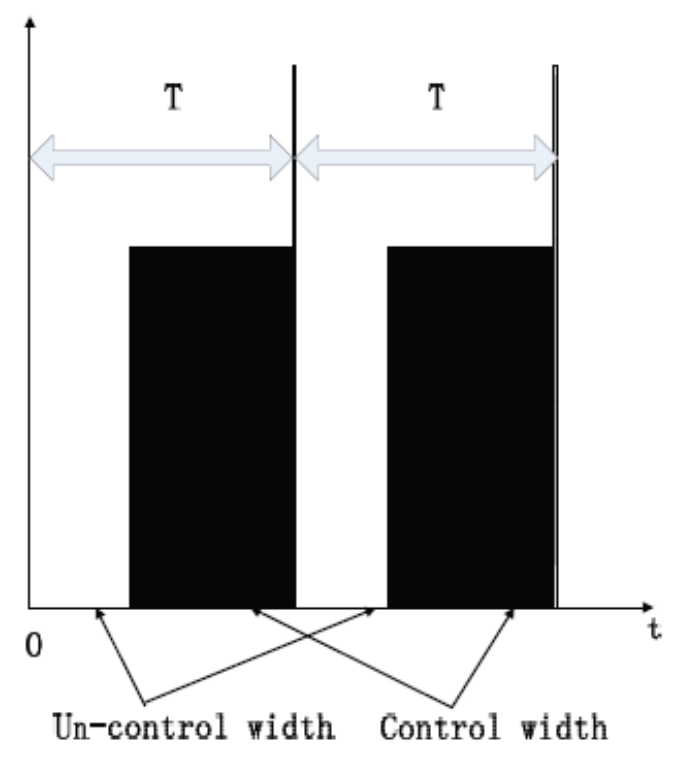

Figure 1: The simplified diagram of the second type intermittent control architecture

To achieve fast synchronization of the error systems (40) via the intermittent controllers (39), we firstly give the following lemma.

Lemma 4. Suppose that function $V(t)$ is continuous and non-negative when $t \in[0, \infty)$ and satisfy the following conditions:

$$
\left\{\begin{array}{lr}
\dot{V}(t) \leq p_{2} V(t), & l T \leq t<l T+\bar{\theta} T \\
\dot{V}(t) \leq-\alpha V^{\eta}(t)-p_{1} V(t), & l T+\bar{\theta} T \leq t<(l+1) T
\end{array}\right.
$$

where $\alpha>0, T>0, p_{1}>0, p_{2}>00<\eta, \bar{\theta}<1, l \in \iota$ are constants, then the following inequality holds:

$$
\begin{gathered}
V^{1-\eta}(t)<\exp \left\{-(1-\eta) p_{1}(1-\bar{\theta}) t\right\} \exp \left\{(1-\eta) p_{2} \bar{\theta} t\right\}\left[V^{1-\eta}(0)+\frac{\alpha}{p_{1}}-\right. \\
\left.\frac{\alpha}{p_{1}} \exp \left\{(1-\eta) p_{1}(1-\bar{\theta}) t\right\} \exp \left\{-(1-\eta) p_{2} \bar{\theta} t\right\}\right] .
\end{gathered}
$$

Similar to Theorem 1, the following results are obtained, which ensure the finite-time synchronization of the drive system (1) and the response system (2) via the new intermittent control technique.

Theorem 2. Suppose that Assumptions 1 and 2 hold, there exist positive constants $\eta_{1}, \eta_{2}, \ldots, \eta_{N}, \xi, p_{1}, p_{2}$ and a positive definite diagonal matrix 
$P>0$ such that the following conditions hold

$$
\begin{gathered}
{\left[\begin{array}{cr}
\left(-\xi+\frac{c_{2} \exp \{p \tau\}}{2(1-\varepsilon)}\right)\left(I_{N} \otimes I_{n}\right)+\left(I_{N} \otimes P \Theta\right)+ & \frac{c_{2}}{2}\left(B \otimes P \Gamma_{2}\right) \\
c_{1}\left(A \otimes P \Gamma_{1}\right)-\frac{q}{2}\left(I_{N} \otimes P\right) & -\frac{c_{2}}{2}\left(I_{N} \otimes I_{n}\right)
\end{array}\right]<0, \quad(42)} \\
* \\
{\left[\begin{array}{cr}
c_{1}\left(A \otimes P \Gamma_{1}\right)+\left(I_{N} \otimes P \Theta\right)+\left(\frac{c_{2} \exp \{p \tau\}}{2(1-\varepsilon)}-\xi\right. & \\
\left.-\frac{\bar{k}}{\lambda}\right)\left(I_{N} \otimes I_{n}\right)-c_{1}(\Xi \otimes P)+\frac{p}{2}\left(I_{N} \otimes P\right) & \frac{c_{2}}{2}\left(B \otimes P \Gamma_{2}\right) \\
* & -\left(\frac{c_{2}}{2}-\frac{\bar{k}}{\lambda}\right)\left(I_{N} \otimes I_{n}\right)
\end{array}\right]<0} \\
p(1-\bar{\theta})-q \bar{\theta}>0,
\end{gathered}
$$

where $\Xi=\operatorname{diag}\left(\eta_{1}, \eta_{2}, \ldots, \eta_{N}\right), \Theta=\operatorname{diag}\left(\theta_{1}, \theta_{2}, \ldots, \theta_{n}\right)$ and $I_{N}$ is the $N \times N$ identity matrix. Then under the periodically intermittent controllers (39), the error systems (40) can converge to zero in a finite time

$$
t \leq \frac{\ln \left(1+\frac{p V^{\frac{1-\mu}{2}}(0)}{\alpha}\right)}{\frac{1-\mu}{2}(p(1-\bar{\theta})-q \bar{\theta})}=T_{3}
$$

where $V(0)=\sum_{i=1}^{N} e_{i}^{T}(0) P e_{i}(0)+\frac{c_{2} \exp \{p \tau\}}{2(1-\varepsilon)} \sum_{i=1}^{N} \int_{-\tau(0)}^{0} \exp \{p s\} e_{i}^{T}(s) e_{i}(s) \mathrm{d} s$, $e_{i}(0)$ is the initial condition of $e_{i}(t)$.

Proof. Similar to Theorem 1, Lyapunov function construct the same as (22), then we have

$$
\left\{\begin{array}{lr}
\dot{V}(t) \leq q V(t), & l T \leq t<l T+\bar{\theta} T \\
\dot{V}(t) \leq-\alpha V^{\eta}(t)-p V(t), & l T+\bar{\theta} T \leq t<(l+1) T
\end{array}\right.
$$

where $\alpha=\min \left\{2^{\frac{1+\mu}{2}} \bar{k},\left(\frac{c_{2} \exp \{p \tau\}}{2(1-\varepsilon)}\right)^{-\frac{1+\mu}{2}} \bar{k}\right\}, \eta=\frac{1-\mu}{2}$.

From Lemma 4, we have

$$
\begin{gathered}
V^{\frac{1-\mu}{2}}(t)<\exp \left\{-\frac{1-\mu}{2} p(1-\bar{\theta}) t\right\} \exp \left\{\frac{1-\mu}{2} q \bar{\theta} t\right\}\left[V^{\frac{1-\mu}{2}}(0)+\frac{\alpha}{p}-\right. \\
\left.\frac{\alpha}{p} \exp \left\{\frac{1-\mu}{2} p(1-\bar{\theta}) t\right\} \exp \left\{-\frac{1-\mu}{2} q \bar{\theta} t\right\}\right] .
\end{gathered}
$$


By Lemma 1, ones obtain

$$
t \leq \frac{\ln \left(1+\frac{p V^{\frac{1-\mu}{2}}(0)}{\alpha}\right)}{\frac{1-\mu}{2}(p(1-\bar{\theta})-q \bar{\theta})} .
$$

This completes the proof.

Remark 8. From (21) and (46), we can see that if $\widetilde{\theta}=\bar{\theta}>0.5$, then $T_{1}<T_{3}$; if $\tilde{\theta}=\bar{\theta}=0.5$, then $T_{1}=T_{3}$; and if $\tilde{\theta}=\bar{\theta}<0.5$, then $T_{1}>T_{3}$.

\section{Numerical examples}

In this section, we give two examples to demonstrate the effectiveness of the theoretical results for finite-time synchronization of complex dynamical networks via periodically intermittent control. For this purpose, Lorenz system is taken as the node dynamics.

The dynamics of Lorenz system is described as follows:

$$
\dot{s}=f(s)=\left[\begin{array}{c}
\dot{s}_{1} \\
\dot{s}_{2} \\
\dot{s}_{3}
\end{array}\right]=\left[\begin{array}{ccc}
-a & a & 0 \\
c & -1 & 0 \\
0 & 0 & -b
\end{array}\right]\left[\begin{array}{c}
s_{1} \\
s_{2} \\
s_{3}
\end{array}\right]+\left[\begin{array}{c}
0 \\
-s_{1} s_{3} \\
s_{1} s_{2}
\end{array}\right] \triangleq C s+\psi(s) .
$$

As shown in Figure 2, Lorenz system has a chaotic attractor when $a=$ $10, c=30, b=8 / 3$. Note that the states of Lorenz system (49) are bounded with $\left|x_{i 1}\right| \leq 18.6360=r_{1},\left|x_{i 2}\right| \leq 25.3679=r_{2},\left|x_{i 3}\right| \leq 45.9792=r_{3}$. Let $x=\left[x_{1}, x_{2}, x_{3}\right]^{T}, y=\left[y_{1}, y_{2}, y_{3}\right]^{T}, \delta=x-y \triangleq\left[\delta_{1}, \delta_{2}, \delta_{3}\right]^{T}=\left[x_{1}-y_{1}, x_{2}-\right.$ $\left.y_{2}, x_{3}-y_{3}\right]^{T},|\epsilon|=\left[\left|\epsilon_{1}\right|,\left|\epsilon_{2}\right|,\left|\epsilon_{3}\right|\right]^{T}$, then

$$
\psi(x)-\psi(y)=\left[\begin{array}{c}
0 \\
-x_{1} x_{3}+y_{1} y_{3} \\
x_{1} x_{2}-y_{1} y_{2}
\end{array}\right]=\left[\begin{array}{c}
0 \\
-x_{1} \epsilon_{3}-y_{3} \epsilon_{1} \\
x_{1} \epsilon_{2}+y_{2} \epsilon_{1}
\end{array}\right] .
$$

Hence,

$$
\begin{aligned}
& (x-y)^{T}(\psi(x)-\psi(y))=-y_{3} \epsilon_{1} \epsilon_{2}+y_{2} \epsilon_{1} \epsilon_{3} \\
& \leq \frac{1}{2}|\epsilon|^{T}\left[\begin{array}{ccc}
0 & r_{3} & r_{2} \\
r_{3} & 0 & 0 \\
r_{2} & 0 & 0
\end{array}\right]|\epsilon| \triangleq \frac{1}{2}|\epsilon|^{T} R|\epsilon| .
\end{aligned}
$$




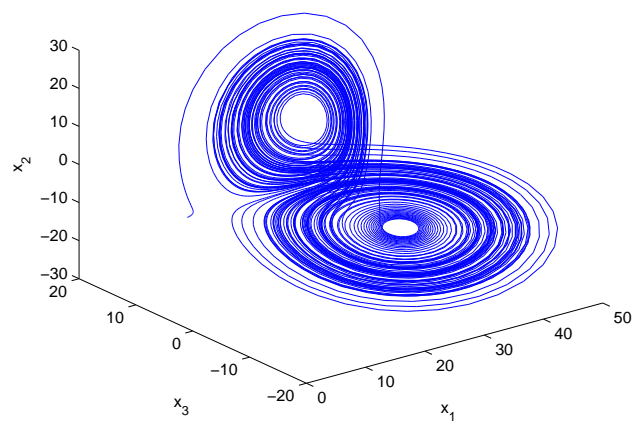

Figure 2: The chaotic trajectories of Lorenz system

Then

$$
\begin{aligned}
& (x-y)^{T} P(f(x)-f(y)-\Theta(x-y)) \leq \\
& \frac{\lambda_{\max }(C)+\frac{1}{2} \lambda_{\max }(R)-\lambda_{\min }(\Theta)}{\lambda_{\min }(P)}(x-y)^{T}(x-y),
\end{aligned}
$$

where $\lambda_{\max }(C), \lambda_{\max }(R), \lambda_{\min }(\Theta)$ are the maximum eigenvalues of $C, R, \Theta$, respectively. Let $\xi=-\frac{\lambda_{\max }(C)+\frac{1}{2} \lambda_{\max }(R)-\lambda_{\min }(\Theta)}{\lambda_{\min }(P)}=38.5143$, therefore Assumption 1 is satisfied with $\Theta=\operatorname{diag}(40,40,40)$ and $P=\operatorname{diag}(0.5,0.4,0.035)$.

Example 1. Consider the following complex dynamical networks (1) consisting of 10 identical Lorenz oscillators nodes, which is described by:

$$
\begin{array}{r}
\dot{x}_{i}(t)=f\left(x_{i}(t)\right)+c_{1} \sum_{j=1}^{10} a_{i j} \Gamma_{1} x_{j}(t)+c_{2} \sum_{j=1}^{10} b_{i j} \Gamma_{2} x_{j}(t-\tau(t)), \\
i=1,2, \cdots, 10 .
\end{array}
$$

Time-varying delay is selected as $\tau(t)=0.01-0.01 \mathrm{e}^{-t}$, then $\tau=0.01, \varepsilon=$ 0.01. And we choose the coupling strengths $c_{1}=10$ and $c_{2}=0.5$, the inner matrices $\Gamma_{1}=\operatorname{diag}(1,1,1), \Gamma_{2}=\operatorname{diag}(5,5,5)$, the non-delayed and time- 
varying delayed weight configuration matrices $A$ and $B$ are given as follows:

$$
\begin{gathered}
A=\left[\begin{array}{cccccccccc}
-1 & 1 & 0 & 0 & 0 & 0 & 0 & 0 & 0 & 0 \\
0 & -2 & 0 & 1 & 0 & 0 & 0 & 0 & 0 & 1 \\
0 & 0 & -1 & 0 & 1 & 0 & 0 & 0 & 0 & 0 \\
0 & 0 & 0 & -1 & 0 & 0 & 0 & 1 & 0 & 0 \\
0 & 1 & 1 & 0 & -3 & 1 & 0 & 0 & 0 & 0 \\
0 & 0 & 0 & 0 & 0 & -1 & 1 & 0 & 0 & 0 \\
0 & 0 & 0 & 0 & 0 & 0 & -1 & 1 & 0 & 0 \\
0 & 0 & 0 & 0 & 0 & 1 & 0 & -2 & 1 & 0 \\
0 & 1 & 0 & 1 & 0 & 0 & 0 & 1 & -3 & 0 \\
0 & 0 & 0 & 0 & 1 & 0 & 0 & 0 & 0 & -1
\end{array}\right], \\
B=\left[\begin{array}{cccccccccc}
-5 & 1 & 1 & 0 & 1 & 0 & 1 & 0 & 1 & 0 \\
1 & -6 & 1 & 0 & 1 & 1 & 1 & 1 & 0 & 0 \\
1 & 1 & -3 & 1 & 0 & 0 & 0 & 0 & 0 & 0 \\
0 & 1 & 1 & -3 & 0 & 1 & 0 & 0 & 0 & 0 \\
1 & 1 & 0 & 0 & -2 & 0 & 0 & 0 & 0 & 0 \\
0 & 1 & 0 & 1 & 0 & -5 & 1 & 2 & 0 & 0 \\
0 & 1 & 0 & 0 & 0 & 1 & -2 & 0 & 0 & 0 \\
0 & 1 & 0 & 0 & 0 & 1 & 0 & -4 & 1 & 1 \\
1 & 0 & 0 & 1 & 0 & 0 & 0 & 1 & -3 & 1 \\
0 & 0 & 0 & 0 & 1 & 0 & 0 & 1 & 1 & -2
\end{array}\right] .
\end{gathered}
$$

The corresponding controlled response system of (2) is described as follows:

$$
\begin{array}{r}
\dot{y}_{i}(t)=f\left(y_{i}(t)\right)+c_{1} \sum_{j=1}^{10} a_{i j} \Gamma_{1} y_{j}(t)+c_{2} \sum_{j=1}^{10} b_{i j} \Gamma_{2} y_{j}(t-\tau(t))+u_{i}(t), \\
i=1,2, \cdots, 10,
\end{array}
$$

where $a_{i j}, b_{i j}$ and $f_{i}(\cdot)$ are the same as (50) and

$$
\left\{\begin{array}{l}
u_{i}(t)=-\eta_{i} e_{i}(t)-\bar{k} \frac{\left(\lambda_{\max }(P)\right)^{\frac{1+\mu}{2}}}{\lambda_{\min }(P)} \operatorname{sign}\left(e_{i}(t)\right)\left|e_{i}(t)\right|^{\mu}, l T \leq t<l T+\delta_{1}, \\
u_{i}(t)=0, \quad l T+\delta_{1} \leq t<(l+1) T
\end{array}\right.
$$

where $\bar{k}=5$.

The initial conditions of the numerical simulations are as follows: $x_{i}(0)=$ $(2+0.2 i, 0.2+0.3 i, 0.3+0.1 i)^{T}, y_{i}(0)=(-8+0.7 i,-5+0.8 i,-10+0.6 i)^{T}$, 


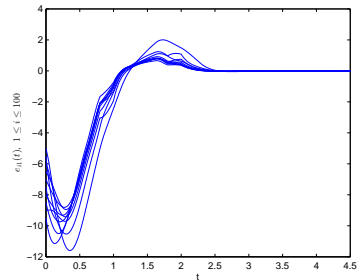

(a) $e_{i 1}$

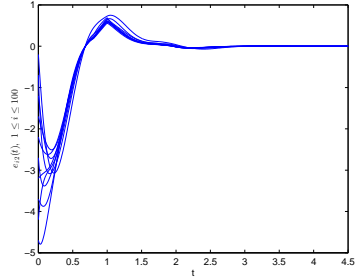

(b) $e_{i 2}$

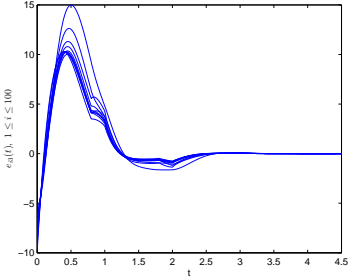

(c) $e_{i 3}$

Figure 3: Synchronization errors $e_{i j}, 1 \leq i \leq 10,1 \leq j \leq 3$ with control parameters $\bar{k}=5, T=1, \widetilde{\theta}=0.8, \mu=0.5, p=0.358, q=1.332$.

where $1 \leq i \leq 10$. The initial conditions of the error system are $e_{i}(0)=$ $(-10+0.5 i,-5.2+0.5 i,-10.3+0.5 i)^{T}$, where $1 \leq i \leq 10$.

In Theorem 1, in order to synchronize the drive system (50) with the response system (51), we select $\Theta=\operatorname{diag}(40,40,40), P=\operatorname{diag}(0.5,0.4,0.035)$, and $\lambda=70$, it is easy to verify that the inequality (18) holds. Besides, to simulate (17) by Matlab LMIs Toolbox, we can obtain the control gain matrix of the intermittent controllers (52) as $\Xi=\operatorname{diag}(39.6586, \ldots, 39.6586)_{10 \times 10}$, $p=0.358$ and $q=1.332$. Choosing the control period $T=1$ and the constant $\mu=0.5$; calculating (20), then $\widetilde{\theta}=0.8$ is satisfied (20). Hence, all the conditions of Theorem 1 are satisfied, which implies that the error systems (4) are finite-time synchronization via intermittent control. The numerical simulations of the error curves are demonstrated in Figure 3. Take $\bar{k}=5, T=$ $1, \widetilde{\theta}=0.8, p=1.424, q=1.332, \mu=0.5$. By simple calculating (21), we have $t=1.98$. The synchronization error variables $e_{i j}, 1 \leq i \leq 10, j=1,2,3$ 5 are showed in the Figure 4 , with $\bar{k}=5, T=1, \widetilde{\theta}=0.8, \mu=0.5, p=$ 6 1.424, $q=1.332$. Let $\bar{k}=5, T=1, \widetilde{\theta}=0.8, p=3.578, q=1.332, \mu=0.5$. Through simple computation (21), we get $t=1.14$. Figure 5 describes the synchronization error variables $e_{i j}, 1 \leq i \leq 10, j=1,2,3$, respectively, with different parameters $p$.

Now we will verify Corollary 1. For convenience, the initial conditions of the numerical simulations are taken as the same as Theorem 1, and let the control rate $\theta=1$. Figure 6 demonstrates the time response of the error variables $e_{i j}, 1 \leq i \leq 10, j=1,2,3$, respectively, with $\bar{k}=5, \widetilde{\theta}=1, \mu=$

$0.5, p=0.358$. The first part of controllers (52) can synchronize the network at about $t=2.15$, which implies that the convergence time is smaller than $t=2.54$ in Theorem 1 . 


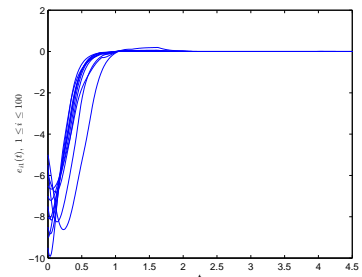

(a) $e_{i 1}$

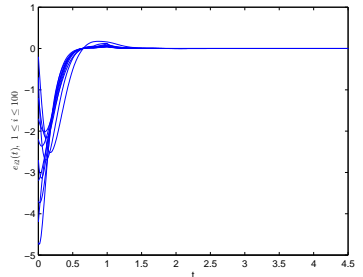

(b) $e_{i 2}$

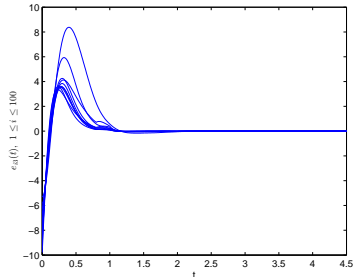

(c) $e_{i 3}$

Figure 4: Synchronization errors $e_{i j}, 1 \leq i \leq 10,1 \leq j \leq 3$ with control parameters $\bar{k}=5, T=1, \tilde{\theta}=0.8, \mu=0.5, p=1.424, q=1.332$.

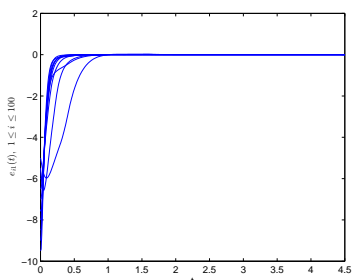

(a) $e_{i 1}$

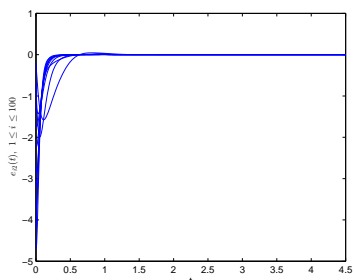

(b) $e_{i 2}$

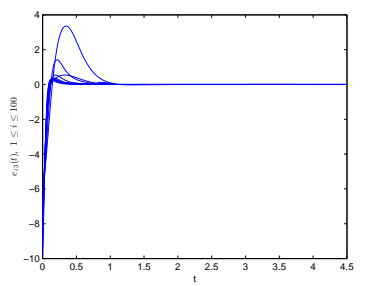

(c) $e_{i 3}$

Figure 5: Synchronization errors $e_{i j}, 1 \leq i \leq 10,1 \leq j \leq 3$ with control parameters $\bar{k}=5, T=1, \widetilde{\theta}=0.8, \mu=0.5, p=3.578, q=1.332$.

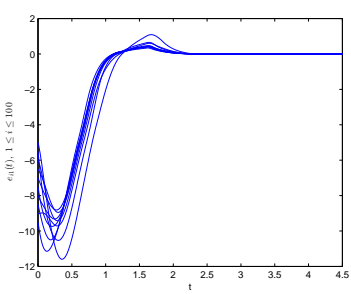

(a) $e_{i 1}$

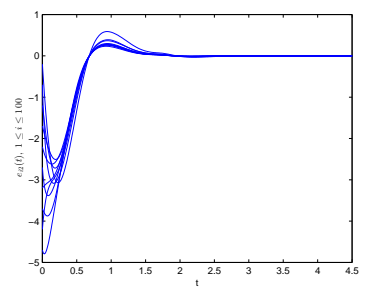

(b) $e_{i 2}$

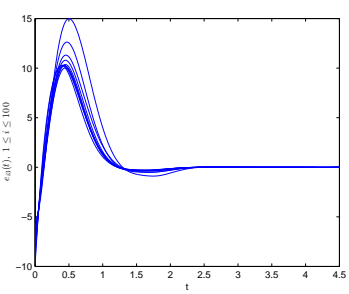

(c) $e_{i 3}$

Figure 6: Synchronization errors $e_{i j}, 1 \leq i \leq 10,1 \leq j \leq 3$ with control parameters $\bar{k}=5, \widetilde{\theta}=1, \mu=0.5, p=0.358$. 


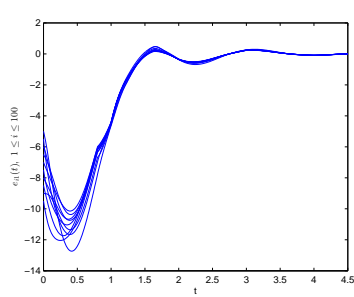

(a) $e_{i 1}$

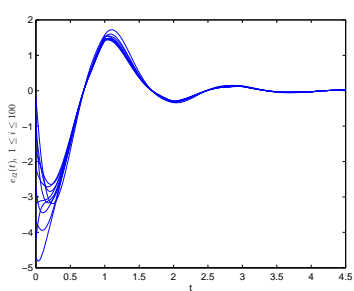

(b) $e_{i 2}$

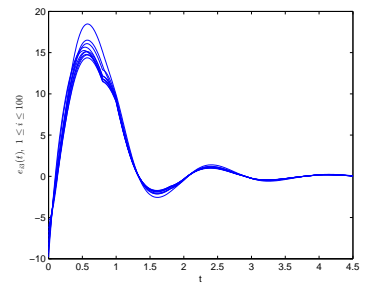

(c) $e_{i 3}$

Figure 7: Synchronization errors $e_{i j}, 1 \leq i \leq 10,1 \leq j \leq 3$ with control parameters $\bar{k}=5, T=1,1-\bar{\theta}=0.2, \mu=0.5, p=3.58, q=1.45$.

Example 2. In this example, we consider the same networks as in Example 1 . We take the control period $T=1$, the control width $1-\bar{\theta}=0.2$ of the intermittent controllers (39) and the other parameters $\bar{k}=5,1-\bar{\theta}=$ $0.2, \mu=0.5, p=3.58, q=1.45$. Figure 7 shows the trajectories of synchronization errors $e_{i j}, 1 \leq i \leq 10, j=1,2,3$, respectively, with $\bar{k}=5, T=$ $1,1-\bar{\theta}=0.2, \mu=0.5, p=3.58, q=1.45$. From Figure 7 , we can find that the networks with intermittent controllers (39) can be synchronized in $t_{1}=3.67($ Figure $6(\mathrm{~m})), t_{1}=3.58($ Figure $6(\mathrm{n}))$, and $t_{1}=3.86($ Figure $6(\mathrm{o}))$, respectively.

\section{Conclusion}

In this paper, the fast synchronization issue of complex dynamical networks via periodically intermittent control is studied. The types of periodically intermittent control scheme are proposed to drive the network achieving finite-time synchronization. Based on the finite-time stability theory and intermittent control techniques, fast synchronization criteria for such dynamical networks are derived by using differential inequalities (14) and (41). Compared with the previous works, our results can achieve fast synchronization with fast convergence time. The proposed conditions (20) in Theorem 1 and (45) in Theorem 2 are required in the intermittent control. If we ignore this condition, then the results of Theorem 1 and Theorem 2 in this paper are not sufficient to be the intermittent control problem. Numerical simulations have proven that the proposed synchronization criteria finally are efficient.

\section{Acknowledgments.}


The authors would like to thank the editor and the anonymous reviewers for their valuable comments and constructive suggestions.

This research is supported by National Natural Science Foundation of China (Grant No.61174216, No. 61273183, No.61374028 and No. 61374085).

\section{Appendix}

Proof of Lemma 2: Take $M_{0}=V^{1-\eta}(0)+\frac{\alpha}{p_{1}}$ and $W(t)=V^{1-\eta}(t) \exp \{(1-$

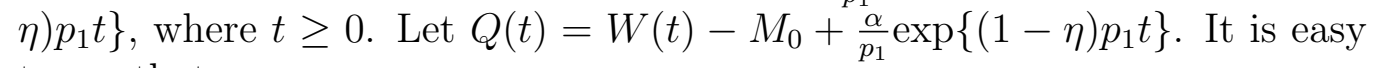
to see that

$$
Q(t)=0, \quad \text { for } t=0 .
$$

In the following, we will prove that

$$
Q(t) \leq 0, \quad \text { for all } t \in[0, \theta T)
$$

If this is not true, then there exists a $t_{1} \in[0, \theta T)$ such that

$$
\begin{array}{ll}
Q\left(t_{1}\right)=0, & \dot{Q}\left(t_{1}\right)>0 \\
Q(t) \leq 0, & 0 \leq t<t_{1} .
\end{array}
$$

Using Eqs. (53), (55) and (56), we obtain

$$
\begin{aligned}
\dot{Q}\left(t_{1}\right)= & (1-\eta) V^{-\eta}\left(t_{1}\right) \dot{V}\left(t_{1}\right) \exp \left\{(1-\eta) p_{1} t_{1}\right\}+ \\
& p_{1}(1-\eta) V^{1-\eta}\left(t_{1}\right) \exp \left\{(1-\eta) p_{1} t_{1}\right\}+\alpha(1-\eta) \exp \left\{(1-\eta) p_{1} t_{1}\right\} \\
\leq & (1-\eta) V^{-\eta}\left(t_{1}\right)\left(-\alpha V^{\eta}\left(t_{1}\right)-p_{1} V\left(t_{1}\right)\right) \exp \left\{(1-\eta) p_{1} t_{1}\right\}+ \\
& p_{1}(1-\eta) V^{1-\eta}\left(t_{1}\right) \exp \left\{(1-\eta) p_{1} t_{1}\right\}+\alpha(1-\eta) \exp \left\{(1-\eta) p_{1} t_{1}\right\} \\
= & -\alpha(1-\eta) \exp \left\{(1-\eta) p_{1} t_{1}\right\}-p_{1}(1-\eta) V^{1-\eta}\left(t_{1}\right) \exp \left\{(1-\eta) p_{1} t_{1}\right\} \\
& +p_{1}(1-\eta) V^{1-\eta}\left(t_{1}\right) \exp \left\{(1-\eta) p_{1} t_{1}\right\}+\alpha(1-\eta) \exp \left\{(1-\eta) p_{1} t_{1}\right\} \\
= & 0 .
\end{aligned}
$$

This contradicts the second inequality in (55), and so (54) holds.

Let $W_{1}(t)=V^{1-\eta}(t) \exp \left\{(1-\eta) p_{1} t\right\} \exp \left\{-(1-\eta) p_{1}(t-\theta T)\right\} \exp \{-(1-$ п) $\left.p_{2}(t-\theta T)\right\}$, and $H(t)=W_{1}(t)-M_{0}+\frac{\alpha}{p_{1}} \exp \left\{(1-\eta) p_{1} t\right\} \exp \left\{-(1-\eta) p_{1}(t-\right.$ $\theta T)\} \exp \left\{-(1-\eta) p_{2}(t-\theta T)\right\}, t \geq \theta T$. Next, we prove that for $t \in[\theta T, T)$,

$$
H(t) \leq 0, \quad \text { for all } t \in[\theta T, T)
$$


Otherwise, there exists a $t_{2} \in[\theta T, T)$ such that

$$
\begin{array}{ll}
H\left(t_{2}\right)=0, & \dot{H}\left(t_{2}\right)>0, \\
H(t) \leq 0, & \theta T \leq t<t_{2} .
\end{array}
$$

According to Eqs. (58) and (59), we have

$$
\begin{aligned}
\dot{H}\left(t_{2}\right)= & \dot{W}_{1}\left(t_{2}\right)+\frac{\alpha}{p_{1}}(1-\eta) p_{1} \exp \left\{(1-\eta) p_{1} t_{2}\right\}\left[\operatorname { e x p } \left\{-(1-\eta) p_{1}\left(t_{2}-\theta T\right)\right.\right. \\
& \exp \left\{-(1-\eta) p_{2}\left(t_{2}-\theta T\right)\right]+\frac{\alpha}{p_{1}} \exp \left\{(1-\eta) p_{1} t_{2}\right\}\left[-(1-\eta)\left(p_{1}+\right.\right. \\
& \left.\left.p_{2}\right) \exp \left\{-(1-\eta)\left(p_{1}+p_{2}\right)\left(t_{2}-\theta T\right)\right\}\right] \\
= & (1-\eta) V^{-\eta}\left(t_{2}\right) \dot{V}\left(t_{2}\right) \exp \left\{(1-\eta) p_{1} t_{2}\right\} \exp \left\{-(1-\eta)\left(p_{1}+p_{2}\right)\left(t_{2}-\right.\right. \\
& \theta T)\}+V^{1-\eta}\left(t_{2}\right)\left[\exp \left\{(1-\eta) p_{1} t_{2}\right\} \exp \left\{-(1-\eta) p_{1}\left(t_{2}-\theta T\right)\right\}\right. \\
& \left.\exp \left\{-(1-\eta) p_{2}\left(t_{2}-\theta T\right)\right\}\right]^{\prime}+\frac{\alpha}{p_{1}}(1-\eta) p_{1} \exp \left\{(1-\eta) p_{1} t_{2}\right\} \\
& \exp \left\{-(1-\eta)\left(p_{1}+p_{2}\right)\left(t_{2}-\theta T\right)\right\}-\frac{\alpha}{p_{1}}(1-\eta)\left(p_{1}+p_{2}\right) \exp \{(1- \\
& \left.\eta) p_{1} t_{2}\right\} \exp \left\{-(1-\eta)\left(p_{1}+p_{2}\right)\left(t_{2}-\theta T\right)\right\} \\
\leq & p_{2}(1-\eta) V^{1-\eta}\left(t_{2}\right) \exp \left\{(1-\eta) p_{1} t_{2}\right\} \exp \left\{-(1-\eta)\left(p_{1}+p_{2}\right)\right. \\
& \left.\left(t_{2}-\theta T\right)\right\}+V^{1-\eta}\left(t_{2}\right)\left[(1-\eta) p_{1} \exp \left\{(1-\eta) p_{1} t_{2}\right\} \exp \{-(1-\right. \\
& \eta)\left(p_{1}+p_{2}\right)\left(t_{2}-\eta\right) p_{2}\left(t_{2}-\theta T\right)-(1-\eta)\left(p_{1}+p_{2}\right) \exp \{(1- \\
& \left.\left.\eta) p_{1} t_{2}\right\} \exp \left\{-(1-\eta)\left(p_{1}+p_{2}\right)\left(t_{2}-\theta T\right)\right\}\right]+\frac{\alpha}{p_{1}}(1-\eta) p_{1} \\
& \exp \left\{(1-\eta) p_{1} t_{2}\right\} \exp \left\{-(1-\eta)\left(p_{1}+p_{2}\right)\left(t_{2}-\theta T\right)\right\}-\frac{\alpha}{p_{1}}(1 \\
& -\eta)\left(p_{1}+p_{2}\right) \exp \left\{(1-\eta) p_{1} t_{2}\right\} \exp \left\{-(1-\eta)\left(p_{1}+p_{2}\right)\left(t_{2}-\theta T\right)\right\} \\
= & p_{2}(1-\eta) V^{1-\eta}\left(t_{2}\right) \exp \left\{(1-\eta) p_{1} t_{2}\right\} \exp \left\{-(1-\eta)\left(p_{1}+p_{2}\right)\left(t_{2}-\right.\right. \\
& \theta T)\}-p_{2}(1-\eta) V^{1-\eta}\left(t_{2}\right) \exp \left\{(1-\eta) p_{1} t_{2}\right\} \exp \left\{-(1-\eta)\left(p_{1}+\right.\right. \\
& \left.\left.p_{2}\right)\left(t_{2}-\theta T\right)\right\}-\frac{\alpha}{p_{1}} p_{2} \exp \left\{(1-\eta) p_{1} t_{2}\right\} \exp \left\{-(1-\eta)\left(p_{1}+p_{2}\right)\left(t_{2}\right.\right. \\
& -\theta T)\} \\
< & 0,
\end{aligned}
$$

which contradicts the second inequality in (58). Hence (57) holds. From 
(57), it is easy to see that

$$
\begin{aligned}
W(t) \leq & \exp \left\{(1-\eta)\left(p_{1}+p_{2}\right)(t-\theta T)\right\}\left[M_{0}-\right. \\
& \left.\frac{\alpha}{p_{1}} \exp \left\{(1-\eta) p_{1} t\right\} \exp \left\{-(1-\eta)\left(p_{1}+p_{2}\right)(t-\theta T)\right\}\right] .
\end{aligned}
$$

Consequently, for $t \in[\theta T, T)$, we have

$$
\begin{aligned}
W(t)< & \exp \left\{(1-\eta)\left(p_{1}+p_{2}\right)(t-\theta T)\right\}\left[M_{0}-\right. \\
& \left.\frac{\alpha}{p_{1}} \exp \left\{(1-\eta) p_{1} t\right\} \exp \left\{-(1-\eta)\left(p_{1}+p_{2}\right)(t-\theta T)\right\}\right] \\
\leq & \exp \left\{(1-\eta)\left(p_{1}+p_{2}\right)(1-\theta) T\right\}\left[M_{0}-\right. \\
& \left.\frac{\alpha}{p_{1}} \exp \left\{(1-\eta) p_{1} t\right\} \exp \left\{-(1-\eta)\left(p_{1}+p_{2}\right)(1-\theta) T\right\}\right] .
\end{aligned}
$$

On the other hand, it follows from Eqs. (53) and (54) that for $t \in[0, \theta T)$,

$$
\begin{aligned}
W(t) \leq & M_{0}-\frac{\alpha}{p_{1}} \exp \left\{(1-\eta) p_{1} t\right\} \\
\leq & M_{0}-\frac{\alpha}{p_{1}} \exp \left\{(1-\eta) p_{1} t\right\} \exp \left\{-(1-\eta)\left(p_{1}+p_{2}\right)(1-\theta) T\right\} \\
\leq & \exp \left\{(1-\eta)\left(p_{1}+p_{2}\right)(1-\theta) T\right\}\left[M_{0}-\right. \\
& \left.\frac{\alpha}{p_{1}} \exp \left\{(1-\eta) p_{1} t\right\} \exp \left\{-(1-\eta)\left(p_{1}+p_{2}\right)(1-\theta) T\right\}\right] .
\end{aligned}
$$

So

$$
\begin{aligned}
W(t)<\exp \left\{(1-\eta)\left(p_{1}+p_{2}\right)(1-\theta) T\right\}\left[M_{0}-\right. \\
\\
\left.\quad \frac{\alpha}{p_{1}} \exp \left\{(1-\eta) p_{1} t\right\} \exp \left\{-(1-\eta)\left(p_{1}+p_{2}\right)(1-\theta) T\right\}\right],
\end{aligned}
$$

for all $t \in[0, T)$.

Similarly, we can proof that for $t \in[T,(1+\theta) T)$,

$$
\begin{aligned}
W(t)< & \exp \left\{(1-\eta)\left(p_{1}+p_{2}\right)(1-\theta) T\right\}\left[M_{0}-\right. \\
& \left.\frac{\alpha}{p_{1}} \exp \left\{(1-\eta) p_{1} t\right\} \exp \left\{-(1-\eta)\left(p_{1}+p_{2}\right)(1-\theta) T\right\}\right],
\end{aligned}
$$


and for $t \in[(1+\theta) T, 2 T)$,

$$
\begin{aligned}
W(t)< & \exp \left\{(1-\eta)\left(p_{1}+p_{2}\right)(1-\theta) T\right\} \exp \left\{(1-\eta)\left(p_{1}+p_{2}\right)(t-\theta T\right. \\
& -T)\}\left[M_{0}-\frac{\alpha}{p_{1}} \exp \left\{(1-\eta) p_{1} t\right\} \exp \left\{-(1-\eta)\left(p_{1}+p_{2}\right)(1\right.\right. \\
& \left.-\theta) T\} \exp \left\{-(1-\eta)\left(p_{1}+p_{2}\right)(t-\theta T-T)\right\}\right] \\
= & \exp \left\{(1-\eta)\left(p_{1}+p_{2}\right)(t-2 \theta T)\right\}\left[M_{0}-\frac{\alpha}{p_{1}}\right. \\
& \left.\exp \left\{(1-\eta) p_{1} t\right\} \exp \left\{-(1-\eta)\left(p_{1}+p_{2}\right)(t-2 \theta T)\right\}\right] .
\end{aligned}
$$

By induction, for any integers $m$, we can derive the following estimation of $W(t)$ for any $m$.

For $m T \leq t<(m+\theta) T$,

$$
\begin{gathered}
W(t)<\exp \left\{(1-\eta)\left(p_{1}+p_{2}\right)(1-\theta) m T\right\}\left[M_{0}-\frac{\alpha}{p_{1}} \exp \{(1\right. \\
\left.\left.-\eta) p_{1} t\right\} \exp \left\{-(1-\eta)\left(p_{1}+p_{2}\right)(1-\theta) m T\right\}\right]
\end{gathered}
$$

and for $(m+\theta) T \leq t<(m+1) T$,

$$
\begin{aligned}
W(t)< & \exp \left\{(1-\eta)\left(p_{1}+p_{2}\right)(t-(m+1) \theta T)\right\}\left[M_{0}-\frac{\alpha}{p_{1}}\right. \\
& \left.\quad \exp \left\{(1-\eta) p_{1} t\right\} \exp \left\{-(1-\eta)\left(p_{1}+p_{2}\right)(t-(m+1) \theta T)\right\}\right] .
\end{aligned}
$$

Since for any $t \geq 0$, there exists a nonnegative integer $k$, such that $k T \leq t<$ $(k+1) T$, we can deduce the following estimation of $W(t)$ for any $t$ by Eqs. (61) and (62).

For $k T \leq t<(k+\theta) T$,

$$
\begin{aligned}
W(t)< & \exp \left\{(1-\eta)\left(p_{1}+p_{2}\right)(1-\theta) k T\right\}\left[M_{0}-\frac{\alpha}{p_{1}}\right. \\
& \left.\exp \left\{(1-\eta) p_{1} t\right\} \exp \left\{-(1-\eta)\left(p_{1}+p_{2}\right)(1-\theta) k T\right\}\right] \\
\leq & \exp \left\{(1-\eta)\left(p_{1}+p_{2}\right)(1-\theta) t\right\}\left[M_{0}-\frac{\alpha}{p_{1}}\right. \\
& \left.\exp \left\{(1-\eta) p_{1} t\right\} \exp \left\{-(1-\eta)\left(p_{1}+p_{2}\right)(1-\theta) t\right\}\right],
\end{aligned}
$$


and for $(k+\theta) T \leq t<(k+1) T$,

$$
\begin{aligned}
W(t)< & \exp \left\{(1-\eta)\left(p_{1}+p_{2}\right)(t-(k+1) \theta T)\right\}\left[M_{0}-\frac{\alpha}{p_{1}} \exp \{(1\right. \\
& \left.\left.-\eta) p_{1} t\right\} \exp \left\{-(1-\eta)\left(p_{1}+p_{2}\right)(t-(k+1) \theta T)\right\}\right] \\
\leq & \left.\exp \left\{(1-\eta)\left(p_{1}+p_{2}\right)(1-\theta) t\right)\right\}\left[M_{0}-\right. \\
& \left.\frac{\alpha}{p_{1}} \exp \left\{(1-\eta) p_{1} t\right\} \exp \left\{-(1-\eta)\left(p_{1}+p_{2}\right)(1-\theta) t\right\}\right] .
\end{aligned}
$$

Together with the definition of $W(t)$, we obtain

$$
\begin{aligned}
& \left.V^{1-\eta}(t) \exp \left\{(1-\eta) p_{1} t\right)\right\} \\
\leq & \exp \left\{(1-\eta)\left(p_{1}+p_{2}\right)(1-\theta) t\right\}\left[V^{1-\eta}(0)+\frac{\alpha}{p_{1}}\right. \\
& \left.-\frac{\alpha}{p_{1}} \exp \left\{(1-\eta) p_{1} \theta t\right\} \exp \left\{-(1-\eta) p_{2}(1-\theta) t\right\}\right] \\
= & \exp \left\{(1-\eta)\left(p_{1}+p_{2}\right)(1-\theta) t\right\}\left[V^{1-\eta}(0)-\frac{\alpha}{p_{1}}\right. \\
& \left.\left(\exp \left\{(1-\eta) p_{1} \theta t\right\} \exp \left\{-(1-\eta) p_{2}(1-\theta) t\right\}-1\right)\right] .
\end{aligned}
$$

It implies the conclusion and the proof is completed.

Proof of Lemma 4. Denote $M_{0}=V^{1-\eta}(0)+\frac{\alpha}{p_{1}}$ and $W(t)=V^{1-\eta}(t) \exp \{-(1-$ 7) $\left.p_{2} t\right\}$, where $t \geq 0$. Let $Q(t)=W(t)-M_{0}+\frac{\alpha}{p_{1}}$. It is easy to see that

$$
Q(t)=0, \quad \text { for } t=0 .
$$

In the following, we will prove that

$$
Q(t) \leq 0, \quad \text { for all } t \in[0, \theta T) .
$$

otherwise, there exists $t_{1} \in[0, \theta T)$ such that

$$
\begin{array}{ll}
Q\left(t_{1}\right)=0, & \dot{Q}\left(t_{1}\right)>0, \\
Q(t)<0, & 0 \leq t<t_{1} .
\end{array}
$$

Using (63), (65) and (65), we have

$$
\begin{aligned}
\dot{Q}\left(t_{1}\right)= & (1-\eta) V^{-\eta}\left(t_{1}\right) \dot{V}\left(t_{1}\right) \exp \left\{-(1-\eta) p_{2} t_{1}\right\}- \\
& (1-\eta) p_{2} V^{1-\eta} \exp \left\{-(1-\eta) p_{2} t_{1}\right\} \\
\leq & p_{2}(1-\eta) V^{-\eta}\left(t_{1}\right) V\left(t_{1}\right) \exp \left\{-(1-\eta) p_{2} t_{1}\right\}- \\
& p_{2}(1-\eta) V^{1-\eta}\left(t_{1}\right) \exp \left\{-(1-\eta) p_{2} t_{1}\right\} \\
= & 0 .
\end{aligned}
$$


This contradicts the second inequality in (65), and so (64) holds.

Let $W_{1}(t)=V^{1-\eta}(t) \exp \left\{-(1-\eta) p_{2} t\right\} \exp \left\{(1-\eta) p_{2}(t-\theta T)\right\} \exp \{(1-$

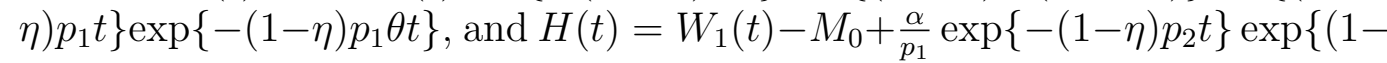
$\left.\eta) p_{2}(t-\theta T)\right\} \exp \left\{(1-\eta) p_{1} t\right\} \exp \left\{-(1-\eta) p_{1} \theta t\right\}, t \geq \theta T$. Next, we prove that for $t \in[\theta T, T)$

$$
H(t) \leq 0, \quad \text { for all } t \in[\theta T, T)
$$

Otherwise, there exists $t_{2} \in[\theta T, T)$ such that

$$
\begin{array}{ll}
H\left(t_{2}\right)=0, & \dot{H}\left(t_{2}\right)>0, \\
H(t)<0, & \theta T \leq t<t_{2} .
\end{array}
$$

According to (68) and (69), we have

$$
\begin{aligned}
\dot{H}\left(t_{2}\right)= & (1-\eta) V^{-\eta}\left(t_{2}\right) \dot{V}\left(t_{2}\right) \exp \left\{-(1-\eta) p_{2} t_{2}\right\} \exp \left\{-(1-\eta) p_{2}\left(t_{2}-\theta T\right)\right\} \\
& \exp \left\{(1-\eta) p_{1} t_{2}\right\} \exp \left\{-(1-\eta) p_{1} \theta t_{2}\right\}-(1-\eta) p_{2} V^{1-\eta}\left(t_{2}\right) \exp \{- \\
& \left.(1-\eta) p_{2} t_{2}\right\} \exp \left\{-(1-\eta) p_{2}\left(t_{2}-\theta T\right)\right\} \exp \left\{(1-\eta) p_{1} t_{2}\right\} \exp \{-(1 \\
& \left.-\eta) p_{1} \theta t_{2}\right\}+V^{1-\eta}\left(t_{2}\right) \exp \left\{-(1-\eta) p_{2} t_{2}\right\}\left(\operatorname { e x p } \left\{-(1-\eta) p_{2}(t-\right.\right. \\
& \left.\theta T)\} \exp \left\{(1-\eta) p_{1} t_{2}\right\}\right)^{\prime} \exp \left\{-(1-\eta) p_{1} \theta t_{2}\right\}+V^{1-\eta}\left(t_{2}\right) \\
& \exp \left\{-(1-\eta) p_{2} t_{2}\right\} \exp \left\{(1-\eta) p_{2}\left(t_{2}-\theta T\right)\right\} \exp \left\{(1-\eta) p_{1} t_{2}\right\} \\
& \left(-\alpha(1-\eta) \frac{p_{2}}{p_{1}}\right) \exp \left\{-(1-\eta) p_{2} t_{2}\right\} \exp \left\{(1-\eta) p_{2}\left(t_{2}-\theta T\right)\right\} \\
& \exp \left\{(1-\eta) p_{1} t_{2}\right\}\left(-(1-\eta) p_{1} \theta\right) \exp \left\{-(1-\eta) p_{1} \theta t_{2}\right\}+ \\
& \alpha(1-\eta) \frac{p_{2}}{p_{1}} \exp \left\{(1-\eta) p_{2} t_{2}\right\} \exp \left\{(1-\eta) p_{2}\left(t_{2}-\theta T\right)\right\} \\
& \exp \left\{(1-\eta) p_{2} t_{2}\right\} \exp \left\{-(1-\eta) p \theta t_{2}\right\}+\frac{\alpha}{p_{1}} \exp \left\{-(1-\eta) p_{2} t_{2}\right\} \\
& \exp \left\{(1-\eta) p_{2}(t-\theta T)\right\}(1-\eta) p_{1} \exp \left\{(1-\eta) p_{1} t_{2}\right\} \exp \{-(1-\eta) \\
& \left.p_{1} \theta t_{2}\right\}+\frac{\alpha}{p_{1}} \exp \left\{(1-\eta) p_{2} t_{2}\right\} \exp \left\{(1-\eta) p_{2} t_{2}\right\}(1-\eta) p_{1} \\
& \exp \left\{(1-\eta) p_{1} t_{2}\right\} \exp \left\{-(1-\eta) p_{1} \theta t_{2}\right\}+\frac{\alpha}{p_{1}} \exp \left\{(1-\eta) p_{2}\left(t_{2}-\theta T\right)\right\} \\
& \exp \left\{(1-\eta) p_{2}\left(t_{2}-\theta T\right)\right\} \exp \left\{(1-\eta) p_{1} t_{2}\right\} \exp \left\{-(1-\eta) p_{1} \theta t_{2}\right\} \\
\leq & 0,
\end{aligned}
$$

which contradicts the second inequality in (68). Hence (67) holds. 
From (67), it is easy to see that

$$
\begin{aligned}
W(t) \leq & \exp \left\{-(1-\eta) p_{2}(t-\theta T)\right\} \exp \left\{-(1-\eta) p_{1} t\right\} \exp \left\{(1-\eta) p_{1} \theta t\right\} \\
& {\left[M_{0}-\frac{\alpha}{p_{1}} \exp \left\{(1-\eta) p_{2}(t-\theta T)\right\} \exp \left\{-(1-\eta) p_{2} t\right\}\right.} \\
& \left.\exp \left\{(1-\eta) p_{1} t\right\} \exp \left\{-(1-\eta) p_{1} \theta t\right\}\right] \\
\leq & \exp \left\{-(1-\eta) p_{2}(t-\theta t)\right\} \exp \left\{-(1-\eta) p_{1} t\right\} \exp \left\{(1-\eta) p_{1} \theta t\right\} \\
& {\left[M_{0}-\frac{\alpha}{p_{1}} \exp \left\{(1-\eta) p_{2}(t-\theta t)\right\} \exp \left\{-(1-\eta) p_{2} t\right\}\right.} \\
& \left.\exp \left\{(1-\eta) p_{1} t\right\} \exp \left\{-(1-\eta) p_{1} \theta t\right\}\right]
\end{aligned}
$$

Consequently, for $t \in[\theta T, T)$, we have

$$
\begin{aligned}
W(t) \leq & \exp \left\{-(1-\eta) p_{2}(t-\theta t)\right\} \exp \left\{-(1-\eta) p_{1}(t-\theta t)\right\}\left[M_{0}-\right. \\
& \left.\frac{\alpha}{p_{1}} \exp \left\{-(1-\eta) p_{2} \theta t\right\} \exp \left\{(1-\eta) p_{1}(t-\theta t)\right\}\right] \\
\leq & M_{0}-\frac{\alpha}{p_{1}} \exp \left\{-(1-\eta) p_{2} \theta t\right\} \exp \left\{(1-\eta) p_{1}(t-\theta t)\right\} \\
\leq & M_{0}-\frac{\alpha}{p_{1}} \exp \left\{-(1-\eta) p_{2} \theta T\right\} \exp \left\{(1-\eta) p_{1}(t-\theta t)\right\} .
\end{aligned}
$$

On the other hand, it follows from Eqs. (63) and (64) that for $t \in[0, \theta T)$,

$$
\begin{aligned}
W(t) & \leq M_{0}-\frac{\alpha}{p_{1}} \\
& \leq M_{0}-\frac{\alpha}{p_{1}} \exp \left\{-(1-\eta) p_{2} \theta T\right\} \exp \left\{(1-\eta) p_{1}(t-\theta t)\right\}
\end{aligned}
$$

From inequalities (72) and (73), we have

$$
W(t) \leq M_{0}-\frac{\alpha}{p_{1}} \exp \left\{-(1-\eta) p_{2} \theta T\right\} \exp \left\{(1-\eta) p_{1}(t-\theta t)\right\},
$$

for $t \in[0, T)$.

Similarly, if $t \in[T,(1+\theta) T)$, we can proof that

$$
\begin{aligned}
W(t) \leq & \left.\exp \left\{-(1-\eta)\left(p_{1}+p_{2}\right)(1-\theta) t\right)\right\} \\
& {\left[M_{0}-\frac{\alpha}{p_{1}} \exp \left\{-(1-\eta) p_{2} \theta t\right\} \exp \left\{(1-\eta) p_{1}(t-\theta t)\right\}\right] }
\end{aligned}
$$


and for $t \in[(1+\theta) T, 2 T)$,

$$
\begin{aligned}
W(t) \leq & \left.\exp \left\{-(1-\eta)\left(p_{1}+p_{2}\right)(1-\theta) t\right)\right\}\left[M_{0}-\frac{\alpha}{p_{1}} \exp \left\{(1-\eta) p_{2}(t\right.\right. \\
& -\theta T)\} \exp \left\{-(1-\eta) p_{2} \theta T\right\} \exp \left\{-(1-\eta) p_{2} t\right\} \\
& \left.\exp \left\{(1-\eta) p_{1}(t-\theta t)\right\}\right] \\
\leq & \left.\exp \left\{-(1-\eta)\left(p_{1}+p_{2}\right)(1-\theta) t\right)\right\}\left[M_{0}-\frac{\alpha}{p_{1}} \exp \left\{(1-\eta) p_{2}(1-\right.\right. \\
& \left.\theta) 2 T)\} \exp \left\{-(1-\eta) p_{2} t\right\} \exp \left\{(1-\eta) p_{1}(t-\theta t)\right\}\right] \\
\leq & \left.\exp \left\{-(1-\eta)\left(p_{1}+p_{2}\right)(1-\theta) t\right)\right\}\left[M_{0}-\frac{\alpha}{p_{1}} \exp \left\{(1-\eta) p_{2}(1-\right.\right. \\
& \left.\theta) t)\} \exp \left\{-(1-\eta) p_{2} t\right\} \exp \left\{(1-\eta) p_{1}(t-\theta t)\right\}\right]
\end{aligned}
$$

Hence, for $\forall t \in[T, 2 T)$, we obtain

$$
\begin{aligned}
W(t) \leq & \left.\exp \left\{-(1-\eta)\left(p_{1}+p_{2}\right)(1-\theta) t\right)\right\} \\
& {\left[M_{0}-\frac{\alpha}{p_{1}} \exp \left\{-(1-\eta) p_{2} \theta t\right\} \exp \left\{(1-\eta) p_{1}(t-\theta t)\right\}\right] }
\end{aligned}
$$

By induction, for any integers $m$, we can derive the following estimation of $W(t)$ for any $m$ and $\forall t \in[m T,(m+1) T)$ such that:

$$
\begin{gathered}
\left.W(t)<\exp \left\{-(1-\eta)\left(p_{1}+p_{2}\right)(1-\theta) t\right)\right\}\left[M_{0}-\frac{\alpha}{p_{1}} \exp \left\{(1-\eta) p_{2}(1-\right.\right. \\
\left.\theta) t\} \exp \left\{-(1-\eta) p_{2} t\right\} \exp \left\{(1-\eta) p_{1}(t-\theta t)\right\}\right]
\end{gathered}
$$

Together with the definition of $W(t)$, we obtain

$$
\begin{aligned}
V^{1-\eta}(t)< & \exp \left\{-(1-\eta) p_{1}(1-\theta) t\right\} \exp \left\{(1-\eta) p_{2} \theta t\right\}\left[V^{1-\eta}(0)+\frac{\alpha}{p_{1}}-\right. \\
& \left.\frac{\alpha}{p_{1}} \exp \left\{(1-\eta) p_{1}(1-\theta) t\right\} \exp \left\{-(1-\eta) p_{2} \theta t\right\}\right] .
\end{aligned}
$$

This completes the proof of Lemma 4.

\section{Reference}

[1] S.H. Strogatz, Nature 410 (2001) 268.

2] M.E.J. Newman, SIAM Rev. 45 (2003) 167.

[3] S. Boccaletti, V. Latora, Y. Moreno, M. Chavez, D.-U. Hwang, Phys. Rep. 424 (2006) 175.

[4] J.A.K. Suykens, G.V. Osipov, Chaos 18 (2008) 037101. 
[5] J. Mei, M. Jiang, Z. Huang, Outer synchronization between two complex networks with identical and nonidentical topological structures, 2011 Fourth International Workshop on Advanced Computational Intelligence (IWACI), (2011): 757-762.

[6] X. Tran, H. Kang, Robust adaptive chatter-free finite-time control method for chaos control and (anti-) synchronization of uncertain (hyper) chaotic systems, Nonlinear Dyn., 80 (2015): 637-651.

[7] S. Jiang, G. Cai, S. Cai, L. Tian, X. Lu, Adaptive cluster general projective synchronization of complex dynamic networks in finite time, Commun Nonlinear Sci Numer Simulat, 28 (2015): 194-200.

[8] M. Jiang, S. Wang, J. Mei, Y. Shen, Finite-time synchronization control of a class of memristor-based recurrent neural networks, Neural Networks, 63 (2015): 133-140.

[9] M. Jiang, J. Mei, J. Hu, New results on exponential synchronization of memristor-based chaotic neural networks, Neurocomputing, 156 (2015) 60-67.

[10] T. Chen, X. Liu, W. Lu, Pinning complex networks by a single controller, IEEE Trans. Circuits Syst. I., 54 (2007): 1317-1326.

[11] W. Wu, W. Zhou, T. Chen, Cluster synchronization of linearly coupled complex networks under pinning control, IEEE Trans. Circuits Syst. I., 56 (2009): 829-839.

[12] Q. Zhang, J. Lu, J. Zhao, Impulsive synchronization of general continuous and discrete-time complex dynamical networks, Commun Nonlinear Sci Numer Simulat, 15(2010): 1063-1070.

[13] X. Liu, K. Zhang, Impulsive control for stabilisation of discrete delay systems and synchronisation of discrete delay dynamical networks, IET Control Theory Appl., 8 (2014): 1185-1195.

[14] Y. Fan, H. Liu, J. Mei, Semi-global finite-time synchronization of complex dynamical networks via periodically intermittent control, Abstract and Applied Analysis, (2015): 381078.

[15] N. Li, H. Sun, X. Jing, Q. Zhang, Exponential synchronisation of united complex dynamical networks with multi-links via adaptive periodically intermittent control, IET Control Theory Appl., 7 (2013): 1725-1736.

[16] J. Huang, C. Li, Q. Han, Stabilization of delayed chaotic neural networks by periodically intermittent control, Circuits Syst Signal Process, 28 (2009): 567-579.

[17] J. Yu, C. Hu, H. Jiang, Z. Teng, Synchronization of nonlinear systems with delays via periodically nonlinear intermittent control, Commun Nonlinear Sci Numer Simulat, 17(2012): 2978-2989. 
[18] H. Zhu, B. Cui, Stabilization and synchronization of chaotic systems via intermittent control, Commun Nonlinear Sci Numer Simulat, 15(2010): $3577-3586$.

[19] J. Mei, M. Jiang, B. Wang, Q. Liu, W. Xu, T. Liao, Exponential psynchronization of non-autonomous Cohen-Grossberg neural networks with reaction-diffusion terms via periodically intermittent control, Neural Processing Letters, 40 (2014): 103-126.

[20] J. Mei, M. Jiang, W. Xu, B. Wang, Finite-time synchronization control of complex dynamical networks with time delay, Commun Nonlinear Sci Numer Simulat, 18 (2013): 2462-2478.

[21] J. Mei, M. Jiang, X. Wang, J. Han, S. Wang, Finite-time synchronization of drive-response systems via periodically intermittent adaptive control, Journal of The Franklin Institute, 351 (2014): 2691-2710.

[22] M.P. Aghababa, S. Khanmohammadi, G, Alizadeh, Finite-time synchronization of two different chaotic systems with unknown parameters via sliding mode technique, Applied Mathematical Modelling, 35 (2011): 3080-3091.

[23] J. Mei, M. Jiang, Z. Wu, X. Wang, Periodically intermittent controlling for finite-time synchronization of complex dynamical networks, Nonlinear Dyn, 79 (1) (2014) 295-305.

[24] J. Mei, M. Jiang, B. Wang, B. Long, Finite-time parameter identification and adaptive synchronization between two chaotic neural networks, Journal of The Franklin Institute, 350 (2013): 1617-1633.

[25] J. Mei, M. Jiang, J. Wang, Finite-time structure identification and synchronization of drive-response systems with uncertain parameter, Commun Nonlinear Sci Numer Simulat, 18 (2013): 999-1015.

[26] X. Liu, N. Jiang, J. Cao, S. Wang, Z. Wang, Finite-time stochastic stabilization for BAM neural networks with uncertainties, Journal of The Franklin Institute, 350 (2013): 2109-2123.

[27] X. Yang, J. Cao, Finite-time stochastic synchronization of complex networks, Appl. Math. Modeling, 34 (2010): 3631-3641.

[28] W. Cui, J. Fang, W. Zhang, X. Wang, Finite-time cluster synchronisation of Markovian switching complex networks with stochastic perturbations, IET Control Theory Appl., 8 (2014): 30-41.

[29] S. Yu, X. Yu, B. Shirinzadeh, Z. Man, Continuous finite-time control for robotic manipulators with terminal sliding mode, Automatica, 41 (2005): 1957-1964.

[30] Y. Shen, Y. Huang, Uniformly observable and globally lipschitzian nonlinear systems admit global finite-time observers, IEEE Trans. Autom. Control, 54 (2009): 2621-2625. 
[31] S. cheng, J. Ji, J. Zhou, Fast synchronization of directionally coupled chaotic systems, Applied Mathematical Modelling, 37 (2013): 127-136.

[32] I. Prodan, E. Zio, F. Stoican, Fault tolerant predictive control design for reliable microgrid energy management under uncertainties, Energy, 91 (2015): 20-34.

[33] K. Ouari, T. Rekioua, M. Ouhrouche, Real time simulation of nonlinear generalized predictive control for wind energy conversion system with nonlinear observer, ISA Transactions, 53 (2014): 76-84.

[34] L. Xu, X. Wang, Mathematical Analysis Methods and Example, Higher Education Press, 1983.

[35] G. E. Escriv, I. S. Heras, M. A. Ortega, Application of an energy management and control system to assess the potential of different control strategies in HVAC systems, Energy and Buildings, 42 (2010): 22582267.

[36] C.B. Chiou, C.H. Chiou, C.M. Chu, S.L. Lin, The study of energysaving strategy for direct expansion air conditioning system, Energy and Buildings, 40 (2008): 1660-1665.

[37] J. Ma, S. Joe Qin, T. Salsbury, Application of economic MPC to the energy and demandminimization of a commercial building, Journal of Process Control, 24 (2014): 1282-1291. 